Nevada

Environmental

Restoration

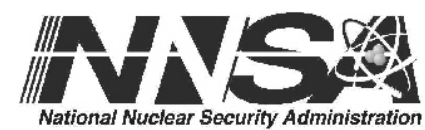

Project

Streamlined Approach for

Environmental Restoration Plan for

Corrective Action Unit 574:

Neptune

Nevada National Security Site,

Nevada

Controlled Copy No::

Revision: 0

August 2011

Environmental Restoration

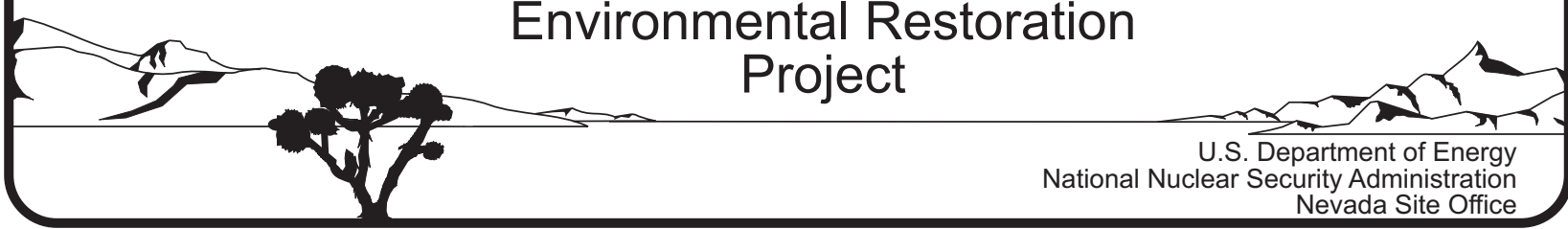




\section{DISCLAIMER}

Reference herein to any specific commercial product, process, or service by trade name, trademark, manufacturer, or otherwise, does not necessarily constitute or imply its endorsement, recommendation, or favoring by the U.S. Government or any agency thereof.

This report has been reproduced directly from the best available copy.

Available for sale to the public from:

U.S. Department of Commerce

National Technical Information Service

5301 Shawnee Road

Alexandria, VA 22312

Telephone: (800) 553-6847

Fax: (703) 605-6900

E-mail: orders@ntis.gov

Online ordering: http://www.ntis.gov/help/ordermethods.aspx

Available electronically at http://www.osti.gov/bridge.

Available for a processing fee to the U.S. Department of Energy and its contractors, in paper, from:

U.S. Department of Energy

Office of Scientific and Technical Information

P.O. Box 62

Oak Ridge, TN 37831-0062

Telephone: (865) 576-8401

Fax: (865) 576-5728

E-mail: reports@adonis.osti.gov 


\title{
STREAMLINED APPROACH FOR ENVIRONMENTAL RESTORATION PLAN FOR CORRECTIVE ACTION UNIT 574: NEPTUNE, NEVADA NATIONAL SECURITY SITE, NEVADA
}

\author{
U.S. Department of Energy \\ National Nuclear Security Administration \\ Nevada Site Office
}

Controlled Copy No.:

Revision: 0

August 2011 
THIS PAGE INTENTIONALLY LEFT BLANK 


\section{STREAMLINED APPROACH FOR ENVIRONMENTAL RESTORATION PLAN FOR CORRECTIVE ACTION UNIT 574: NEPTUNE \\ NEVADA NATIONAL SECURITY SITE, NEVADA}

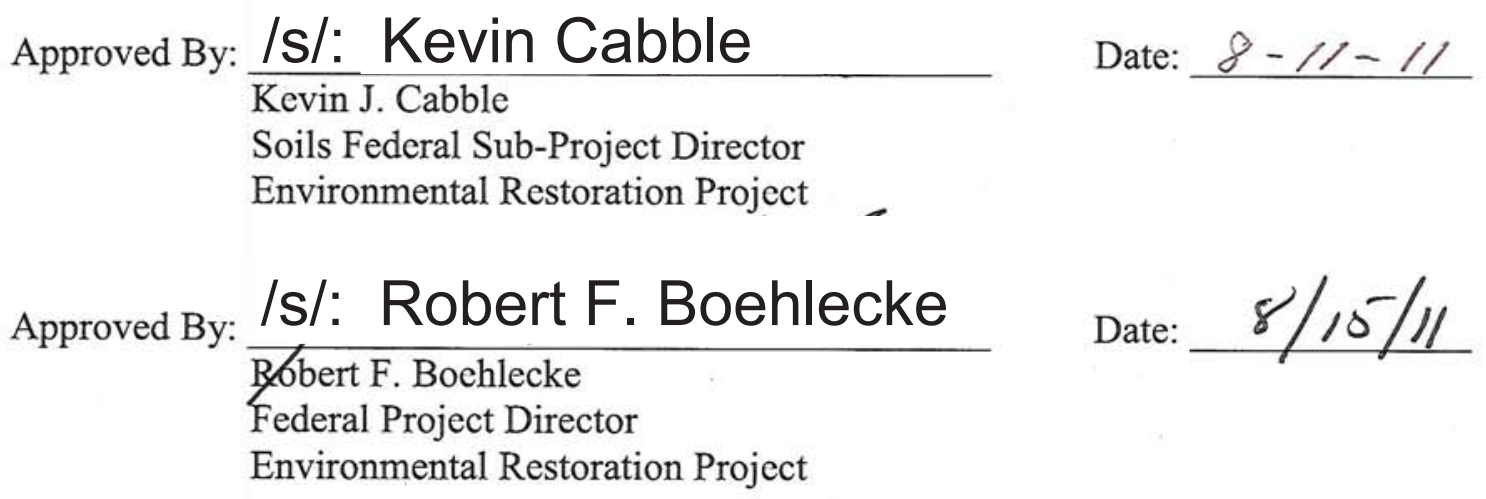


THIS PAGE INTENTIONALLY LEFT BLANK 


\section{TABLE OF CONTENTS}

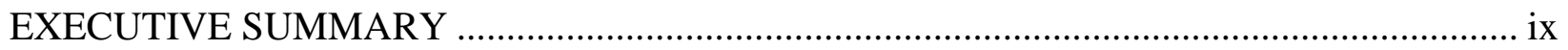

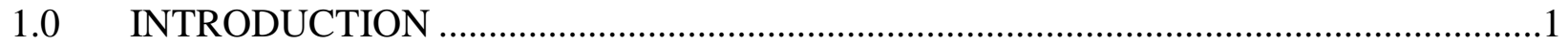

1.1 SAFER PROCESS ...........................................................................................

1.2 SUMMARY OF PROPOSED CORRECTIVE ACTIONS .......................................................4

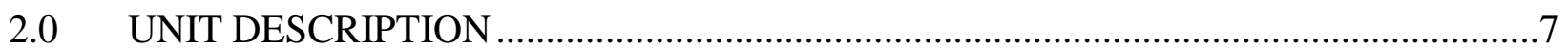

2.1 CoRrective Action Site 12-23-10, U12C.03 Crater (NEPTUNE) .........................

2.1.1 History and Process Knowledge ...................................................................

2.1.2 Physical Setting.....................................................................................

2.1.3 Site Access and Use .........................................................................10

2.1.4 Available Characterization Information ..................................................11

2.1.4.1 Aerial Radiological (Flyover) Survey Data ...................................11

2.1.4.2 Corrective Action Unit 551 Characterization Data......................15

2.1.4.3 Radionuclide Inventory and Distribution Program Data ..............15

2.2 Corrective Action Site 12-45-01, U12E.05 Crater (BlanCA) .........................23

2.2.1 History and Process Knowledge ..............................................................23

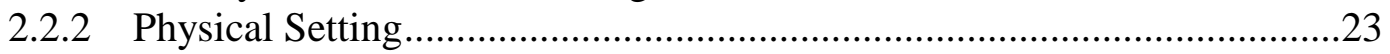

2.2.3 Site Access and Use ..........................................................................23

2.2.4 Available Characterization Information ...................................................25

2.2.4.1 Aerial Radiological (Flyover) Survey Data ..................................25

2.2.4.2 Radionuclide Inventory and Distribution Program Data .............25

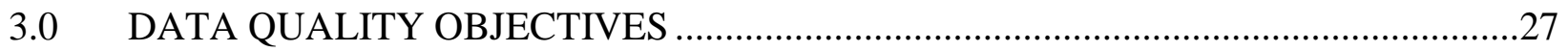

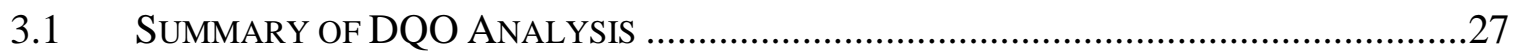

3.2 RESULTS OF THE DQO ANALYSIS ....................................................................28

3.2.1 Action Level Determination and Basis ....................................................28

3.2.2 Hypothesis Test.....................................................................................32

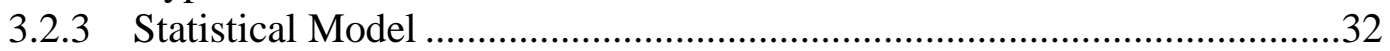

3.2.4 Design Description/Option ......................................................................32

3.2.5 Conceptual Site Model..............................................................................32

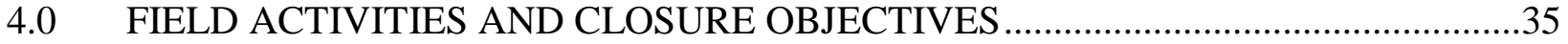

4.1 Contaminants OF Potential CONCERn...............................................................35

4.1.1 Corrective Action Site 12-23-10, U12c.03 Crater (Neptune) ......................35

4.1.2 Corrective Action Site 12-45-01, U12e.05 Crater (Blanca) ........................35

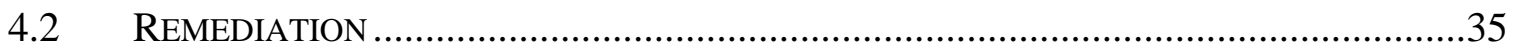

4.2.1 Corrective Action Site 12-23-10, U12c.03 Crater (Neptune) ......................35

4.2.1.1 Calculate External Dose Rates ......................................................35

4.2.1.2 Calculate Internal Dose Rates ......................................................36

4.2.1.3 Define Use Restriction Boundaries...............................................36

4.2.2 Corrective Action Site 12-45-01, U12e.05 Crater (Blanca) .........................36

4.2.2.1 Collect In Situ External Dose Rate Measurements.......................37

4.2.2.2 Calculate Internal Dose Rates ......................................................37

4.2.2.3 Define Use Restriction Boundaries..............................................37

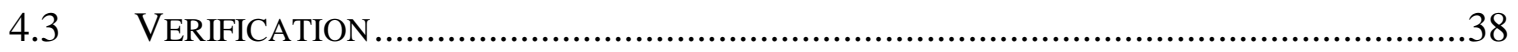

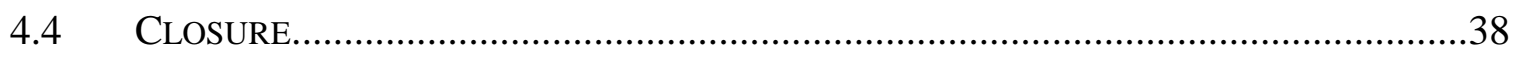




\section{TABLE OF CONTENTS (continued)}

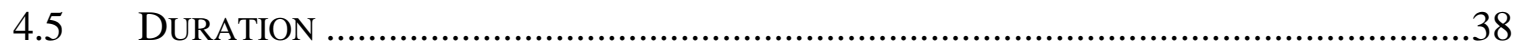

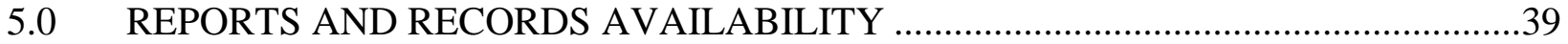

6.0 INVESTIGATION/REMEDIATION WASTE MANAGEMENT .....................................41

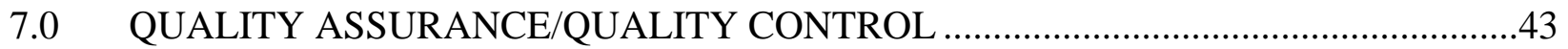

7.1 SAMPLE COLLECTION ACTIVITIES.........................................................................43

7.2 APPLICABLE LABORATORY/ANALYTICAL DATA QUALITY INDICATORS....................43

7.2.1 Precision...........................................................................................44

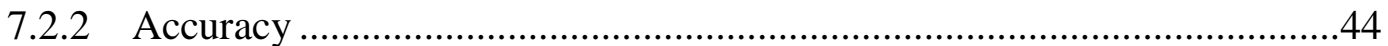

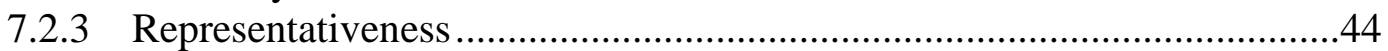

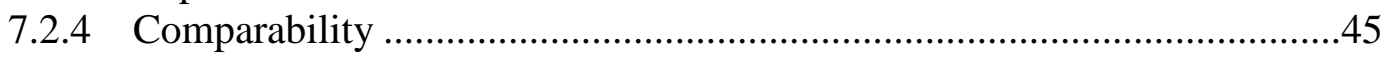

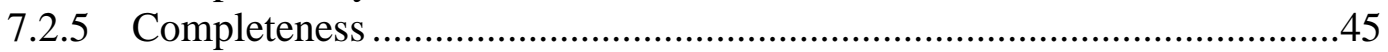

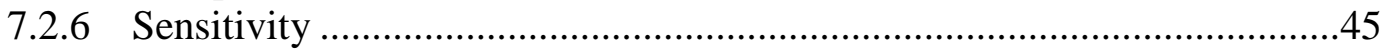

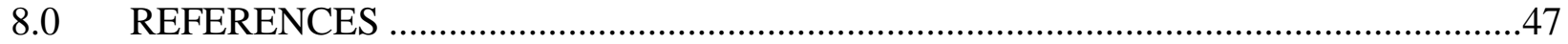

LIBRARY DISTRIBUTION LIST

\section{LIST OF FIGURES}

Figure 1. Corrective Action Unit 574 Site Location MaP ……........................................... 2

Figure 2. CoRRECtive Action Site LoCATION MAP.......................................................... 3

Figure 3. Corrective Action Unit 574 Closure Decision Process ............................................ 5

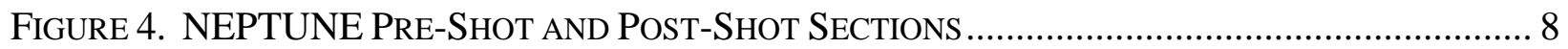

FIGURE 5. NEPTUNE CRATER LOCATION, 10/14/1958_.......................................................... 10

FIGURE 6. VIEW FROM RIM OF NEPTUNE CRATER, 10/23/1958................................................ 10

Figure 7. NEPTUNE CRATER AND Post-Shot Damage, 10/23/1958 ….................................... 11

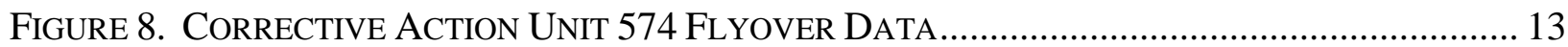

Figure 9. CORRECTIVE ACTION UNIT 551 SOIL SAMPLE LOCATIONS AND USE RESTRICTION

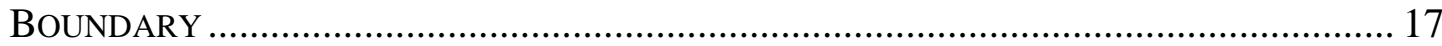

FigURE 10. BLANCA DETONATION, 10/30/1958 .................................................................... 24

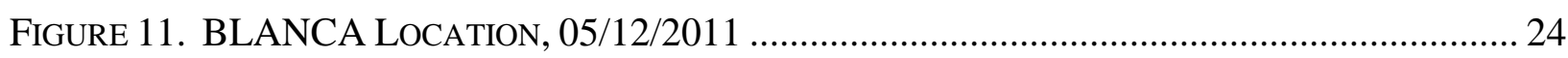

Figure 12. GoOgLe Earth TerRain View of BLANCA AREA .................................................... 25

\section{LIST OF TABLES}

TABLE 1. Corrective Action Unit 551 SoIL SAMPLE Results..................................................... 19

Table 2. Residual Radioactive Material Guidelines Derived for

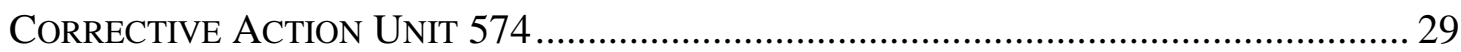

Table 3. Residual Radioactive Computer Code InPut Parameters ...................................... 30

TABle 4. Conceptual Site Model for CoRrective Action Unit 574 ...................................... 33

TABle 5. Project SChedule For Corrective Action Unit 574 ............................................... 38 


\section{TABLE OF CONTENTS (continued)}

APPENDICES

Appendix A. Project Organization

Appendix B. Evaluation of Corrective Action Alternatives 
Revision: 0

Date: August 2011

\section{THIS PAGE INTENTIONALLY LEFT BLANK}




\section{ACRONYMS AND ABBREVIATIONS}

\begin{tabular}{|c|c|}
\hline amsl & above mean sea level \\
\hline ANPR & Advance Notice of Proposed Rulemaking \\
\hline bgs & below ground surface \\
\hline CA & Contamination Area \\
\hline CAA & corrective action alternative \\
\hline CAI & corrective action investigation \\
\hline CAS & Corrective Action Site \\
\hline CAU & Corrective Action Unit \\
\hline CFR & Code of Federal Regulations \\
\hline $\mathrm{cm}$ & centimeter(s) \\
\hline COPC & contaminant of potential concern \\
\hline $\mathrm{CR}$ & Closure Report \\
\hline CSM & conceptual site model \\
\hline $\mathrm{CZ}$ & contamination zone \\
\hline DOE & U.S. Department of Energy \\
\hline $\mathrm{DOE} / \mathrm{NV}$ & U.S. Department of Energy, Nevada Operations Office \\
\hline DQI & data quality indicator \\
\hline DQO & data quality objective \\
\hline DRI & Desert Research Institute \\
\hline EPA & U.S. Environmental Protection Agency \\
\hline FAL & final action level \\
\hline FFACO & Federal Facility Agreement and Consent Order \\
\hline $\mathrm{ft}$ & foot (feet) \\
\hline $\mathrm{g} / \mathrm{cm}^{3}$ & gram(s) per cubic centimeter \\
\hline $\mathrm{g} / \mathrm{m}^{3}$ & gram(s) per cubic meter \\
\hline $\mathrm{g} / \mathrm{yr}$ & gram(s) per year \\
\hline LLNL & Lawrence Livermore National Laboratory \\
\hline $\mathrm{m}$ & meter(s) \\
\hline $\mathrm{m}^{2}$ & square meter(s) \\
\hline $\mathrm{m}^{3} / \mathrm{yr}$ & cubic meter(s) per year \\
\hline $\mathrm{m} / \mathrm{s}$ & meter(s) per second \\
\hline $\mathrm{m} / \mathrm{yr}$ & meter(s) per year \\
\hline
\end{tabular}




\begin{tabular}{ll} 
ACRONYMS AND ABBREVIATIONS (continued) \\
\hline MDC & minimum detectable concentration \\
mg/day & milligram(s) per day \\
mrem/yr & millirem(s) per year \\
N/A & not applicable \\
NAC & Nevada Administrative Code \\
NCRP & National Council on Radiation Protection \\
NDEP & Nevada Division of Environmental Protection \\
NNSA/NSO & U.S. Department of Energy, National Nuclear Security Administration \\
& Nevada Site Office \\
NNSS & Nevada National Security Site \\
NSTec & National Security Technologies, LLC \\
pCi/g & picocurie(s) per gram \\
RCRA & Resource Conservation and Recovery Act \\
RESRAD & Residual Radioactive \\
RIDP & Radionuclide Inventory and Distribution Program \\
RRMG & Residual Radioactive Material Guideline \\
SAFER & Streamlined Approach for Environmental Restoration \\
TED & total effective dose \\
TLD & thermoluminescent dosimeter \\
UR & use restriction \\
USGS & U.S. Geological Survey \\
yr & year(s) \\
& \\
\hline
\end{tabular}




\section{EXECUTIVE SUMMARY}

This Streamlined Approach for Environmental Restoration (SAFER) Plan identifies the activities required for closure of Corrective Action Unit (CAU) 574, Neptune. CAU 574 is included in the Federal Facility Agreement and Consent Order (FFACO) (1996 [as amended March 2010]) and consists of the following two Corrective Action Sites (CASs) located in Area 12 of the Nevada National Security Site:

- CAS 12-23-10, U12c.03 Crater (Neptune)

- CAS 12-45-01, U12e.05 Crater (Blanca)

This plan provides the methodology for the field activities that will be performed to gather the necessary information for closure of the two CASs. There is sufficient information and process knowledge regarding the expected nature and extent of potential contaminants to recommend closure of CAU 574 using the SAFER process. Based on historical documentation, personnel interviews, site process knowledge, site visits, photographs, field screening, analytical results, the results of the data quality objective (DQO) process (Section 3.0), and an evaluation of corrective action alternatives (Appendix B), closure in place with administrative controls is the expected closure strategy for CAU 574.

Additional information will be obtained by conducting a field investigation to verify and support the expected closure strategy and provide a defensible recommendation that no further corrective action is necessary. This will be presented in a Closure Report that will be prepared and submitted to the Nevada Division of Environmental Protection (NDEP) for review and approval.

The sites will be investigated based on the DQOs developed on May 23, 2011, by representatives of NDEP; the U.S. Department of Energy, National Nuclear Security Administration Nevada Site Office; National Security Technologies, LLC; and Navarro-Intera. The DQO process has been used to identify and define the type, amount, and quality of data needed to determine and implement appropriate corrective actions for CAU 574.

The following activities will support closure of CAU 574:

- Collect in situ external dose rate measurements using thermoluminescent dosimeters.

- Combine internal and external dose rates to determine whether total effective dose rates exceed the final action level (FAL).

- If contamination exceeds the FAL, define the extent of the contamination exceeding the FAL, establish closure in place as the corrective action, and implement appropriate use restrictions.

This SAFER Plan has been developed in accordance with the FFACO that was agreed to by the State of Nevada; the U.S. Department of Energy, Environmental Management; the U.S. Department of Defense; and the U.S. Department of Energy, Legacy Management. Under the FFACO, this SAFER Plan will be submitted to NDEP for approval. Fieldwork will be conducted following approval of the plan. 
Revision: 0

Date: August 2011

\section{THIS PAGE INTENTIONALLY LEFT BLANK}




\subsection{INTRODUCTION}

This Streamlined Approach for Environmental Restoration (SAFER) Plan identifies the activities required for closure of Corrective Action Unit (CAU) 574, Neptune. CAU 574 is included in the Federal Facility Agreement and Consent Order (FFACO) (1996 [as amended March 2010]), and consists of two Corrective Action Sites (CASs) located in Area 12 of the Nevada National Security Site (NNSS). The two CASs within CAU 574 are shown on Figures 1 and 2 and are listed below:

- CAS 12-23-10, U12c.03 Crater (Neptune)

- CAS 12-45-01, U12e.05 Crater (Blanca)

\subsection{SAFER PROCESS}

CAUs that may be closed using the SAFER process have conceptual corrective actions that are clearly identified, although some degree of investigation may be necessary to affirm the chosen corrective action and provide sufficient data to implement the corrective action. Consequently, corrective action alternatives (CAAs) can be chosen before completing a corrective action investigation (CAI), given anticipated investigation results.

Uncertainty is limited to an acceptable level of risk and is addressed by documented assumptions that are verified by historical documentation, sampling and analysis, data evaluation, and onsite observations. Closure activities may proceed simultaneously with site characterization as sufficient data are gathered to confirm or disprove the assumptions made during selection of the CAA. If, at any time during the closure process, new information is discovered that indicates that closure activities should be revised, corrective actions will be re-evaluated as appropriate.

The SAFER process combines elements of the data quality objective (DQO) process and the observational approach to plan and conduct closure activities. The observational approach provides a framework for managing uncertainty during the planning and decision-making phases of the project. The DQOs are used to identify the problem and define the type and quality of data needed to complete the investigation. The SAFER Plan includes decision points and criteria for making DQO decisions. The DQOs for CAU 574 are described in Section 3.0.

The purpose of the CAI is to verify the adequacy of existing information, affirm the chosen CAA, provide sufficient data to implement corrective actions, and confirm that closure objectives were met. The actual CAA selected will be based on the results of CAI activities.

Historical documentation and existing characterization data were reviewed to develop this SAFER Plan. There is sufficient information to close CAU 574 using the SAFER process. Based on historical documentation, personnel interviews, site process knowledge, site visits, photographs, field screening, analytical results, the results of the DQO process, and an evaluation of CAAs (Appendix B), closure in place with administrative controls is the expected closure strategy for CAU 574. 
Date: August 2011

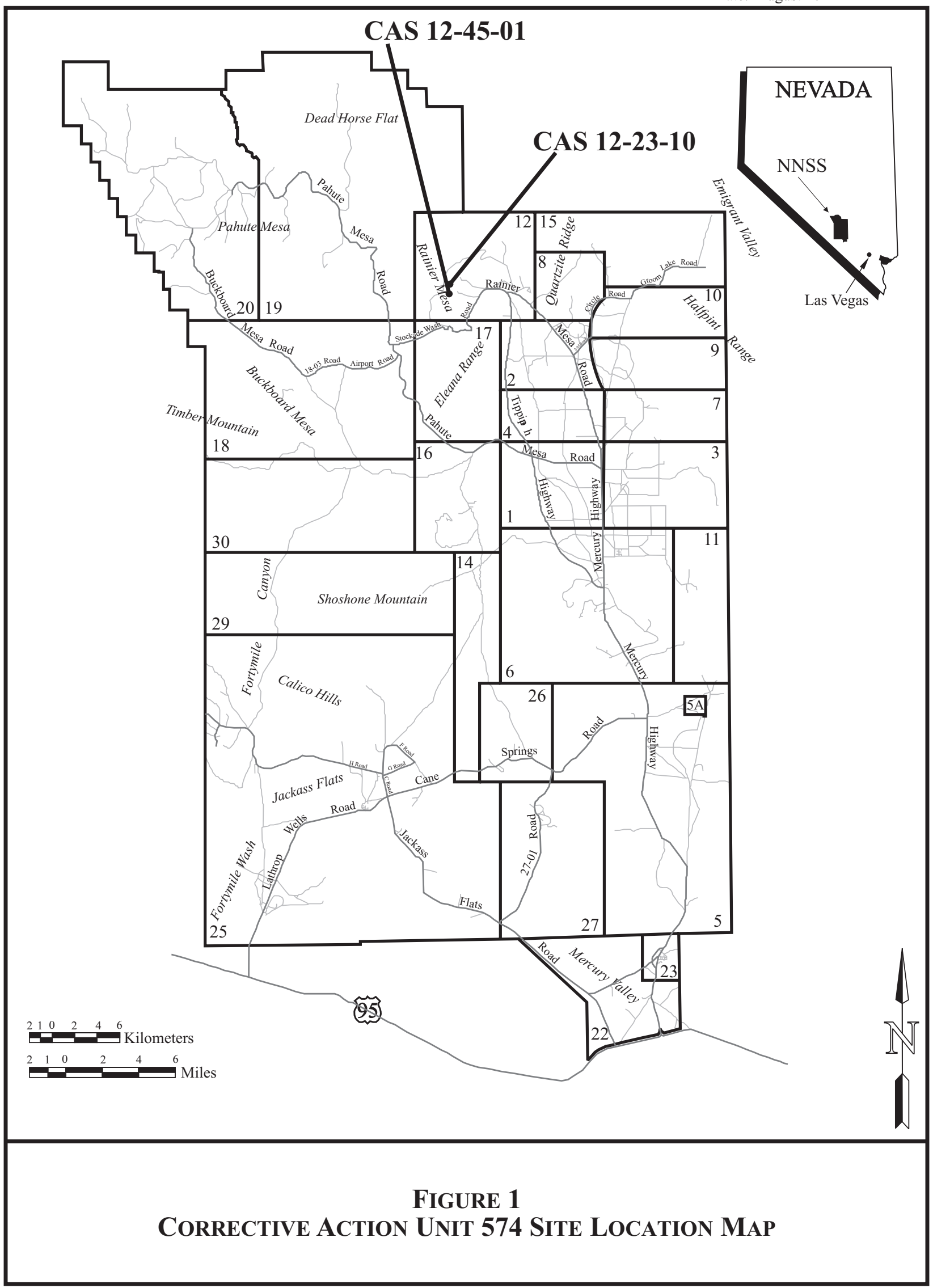




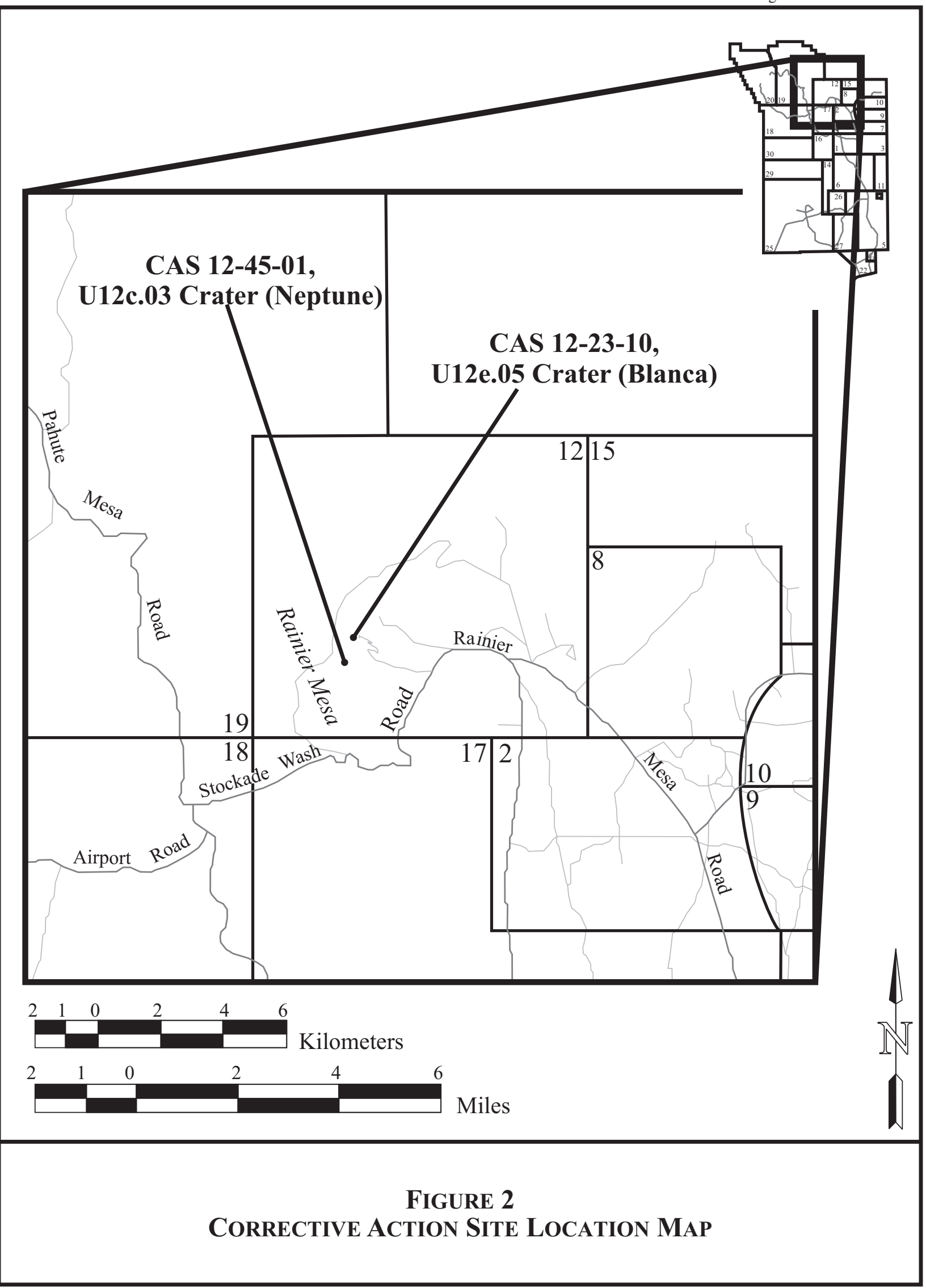




\subsection{Summary of Proposed Corrective Actions}

The decision process for closure of CAU 574 is summarized in Figure 3. If contaminants are present at concentrations above the final action levels (FAL), the extent of contamination will be delineated and closure in place will be implemented. Contingencies are built into the process in the event new information that indicates the selected closure option should be revised is identified. The process ends with closure of the site and preparation of a Closure Report (CR).

Decision points that require a consensus be reached between the U.S. Department of Energy, National Nuclear Security Administration Nevada Site Office (NNSA/NSO) and the Nevada Division of Environmental Protection (NDEP) before continuing are indicated in Figure 3. In addition to these decision points, work may be temporarily suspended until the issue can be satisfactorily resolved if any of the following unexpected conditions occur:

- Conditions outside the scope of work are encountered.

- Unexpected conditions, including unexpected waste and/or contamination, are encountered.

- Unsafe conditions or work practices are identified.

- The conceptual site model (CSM) is shown to be incorrect.

The following activities will support closure of CAU 574:

- Collect in situ external dose rate measurements using thermoluminescent dosimeters (TLDs).

- Combine internal and external dose rates to determine whether total effective dose (TED) rates exceed the FAL.

- If contamination exceeds the FAL, define the extent of the contamination exceeding the FAL, establish closure in place as the corrective action, and implement appropriate use restrictions (URs). 


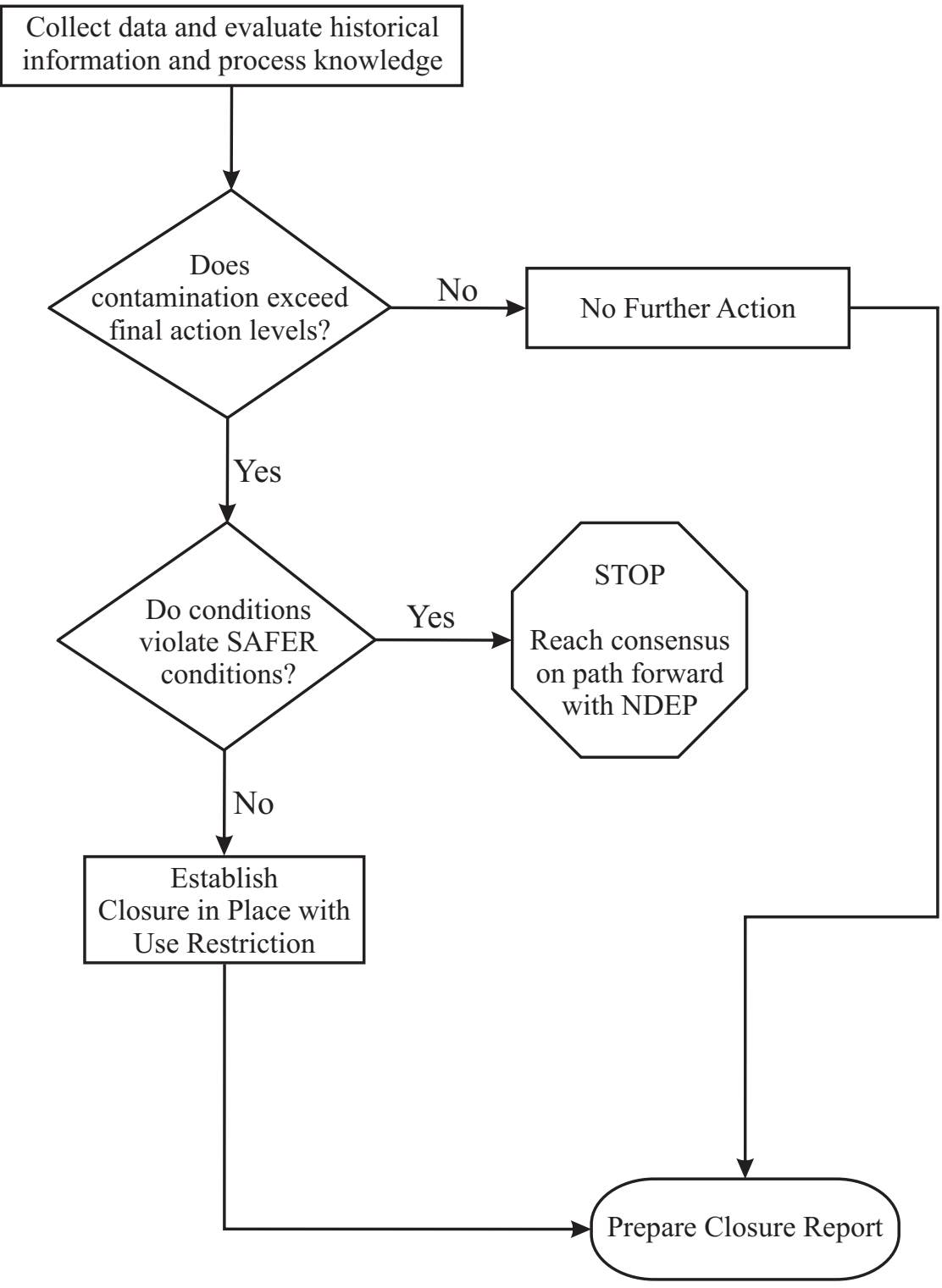

FIGURE 3 Corrective Action Unit 574 Closure Decision Process 
CAU 574 SAFER Plan

Section: Introduction

Revision: 0

Date: August 2011

THIS PAGE INTENTIONALLY LEFT BLANK 


\subsection{UNIT DESCRIPTION}

CAU 574, which includes two CASs in Area 12 of the NNSS, is under the Soils Sub-Project of the NNSA/NSO Environmental Restoration Project. The operational history, process knowledge, and existing information for each CAS are summarized in this section. This information has been obtained through historical document reviews, evaluation of existing data, and interviews with past and present NNSS employees.

Based on process knowledge and historical information, assumptions were made to formulate a CSM to describe the most probable scenario for the current conditions at each CAS. Historical information on the testing that resulted in the CAU 574 CASs shows that a distinct, test-related release of radionuclides occurred that could lead to a total worker dose greater than 25 millirems per year (mrem/yr). The CSM is based on process knowledge and historical data that indicate that radiological contamination resulted from the surface deposition of radionuclides. Section 3.2.5 provides additional information on the CSM developed for CAU 574.

\subsection{Corrective Action Site 12-23-10, U12c.03 Crater (NePTUNE)}

CAS 12-23-10 (referred to as NEPTUNE in this document) consists of soil contamination associated with the NEPTUNE underground nuclear detonation.

\subsubsection{History and Process Knowledge}

The NEPTUNE detonation, conducted on October 14, 1958, was part of Operation Hardtack II. Sponsored by Lawrence Livermore National Laboratory (LLNL), the test was a safety experiment detonated in C-Tunnel, 110 feet (ft) below the surface, and had a yield of approximately 115 tons (U.S. Department of Energy, Nevada Operations Office [DOE/NV], 2000). The surface, located on a 30-degree slope on the side of the Rainier Mesa, bulged upward 15 to $35 \mathrm{ft}$, and a release of radioactive material occurred due to a breach in the ceiling of the tunnel. Material and gas vented with sufficient force to throw very large rocks 80 to $100 \mathrm{ft}$ in the air. Debris formed a slide terminating approximately $800 \mathrm{ft}$ down the slope of the mountain, and a subsidence crater $200 \mathrm{ft}$ in diameter and $35 \mathrm{ft}$ deep was formed (Shelton et al., 1960). Figure 4 shows the pre-shot and post-shot vertical sections normal to the mesa slope contours.

Approximately 1 to 2 percent of the radioactive material vented at the time of crater formation. Levels of radiation in and near the crater 5 days later were less than 100 milliroentgens per hour (Johnson and Violet, 1958). Holes were drilled into the area to determine the physical state of the rock and to delineate the extent of radioactive contamination. Gamma intensity logs of the holes indicate the center of activity is $16 \mathrm{ft}$ below the original zero point (Shelton et al., 1960). It is assumed that subsurface contamination is present in the crater due to direct injection of radionuclides into the subsurface soil from the nuclear test.

Excavated debris and muck generated during tunnel re-entry and recovery activities following the test may have contained radioactive material. Until the mid-1970s, contaminated muck removed during mining and re-entry operations was placed in muckpiles. Clean muck may have then been placed atop contaminated muck. In the mid-1970s, muck determined to be radioactively contaminated was stored in unused underground drifts. Decontamination of equipment may also have occurred on the muckpiles. This practice was common until the mid-1980s (NNSA/NSO, 2004). 


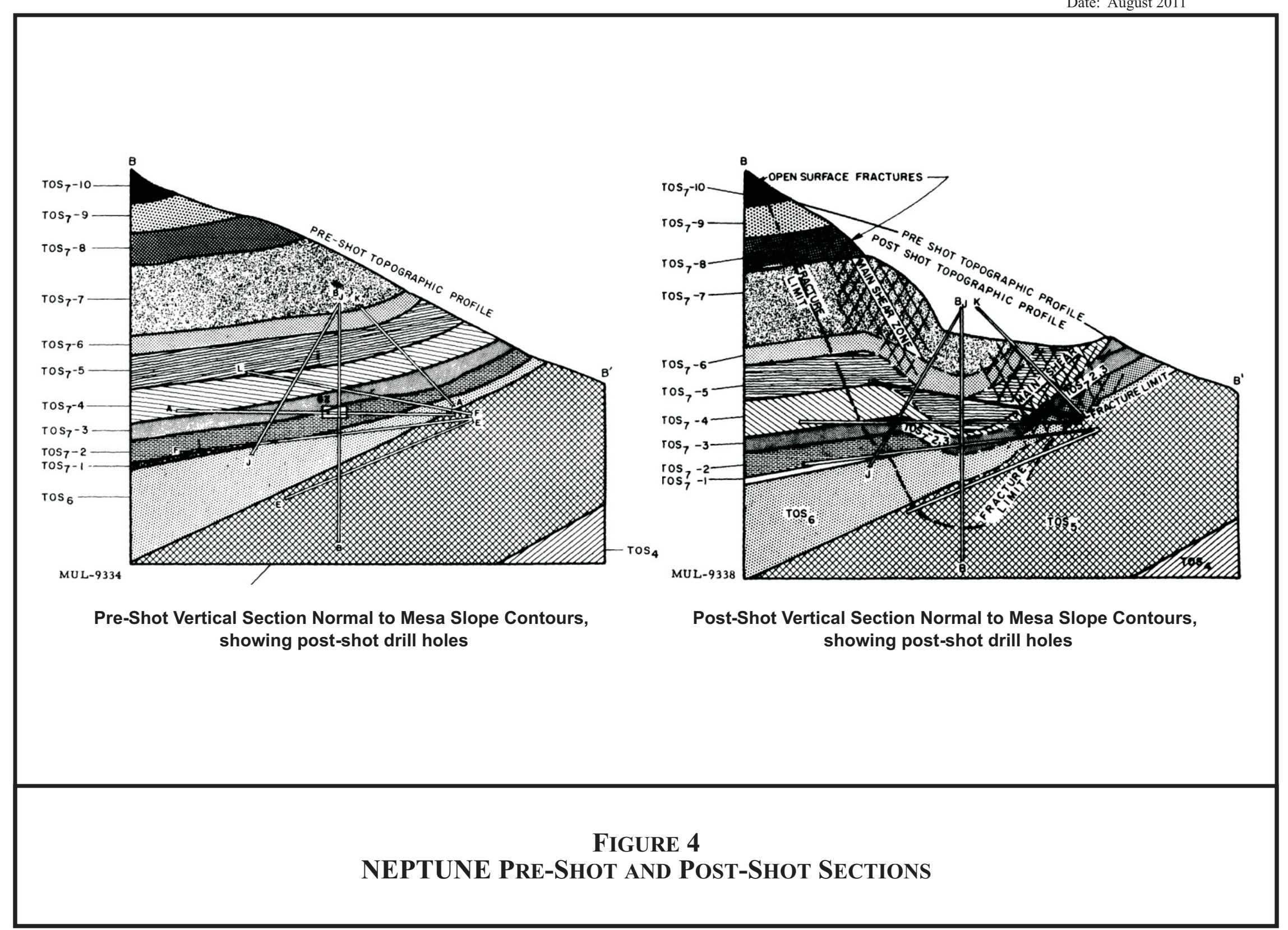


The primary contaminants at this site are plutonium and americium, which contribute to the internal dose component of TED. This is based on both operational history and historical data from this and other safety experiments. Safety experiments result in plutonium and americium contamination because the weapon material (plutonium) is not consumed in the fission process. Historical data collected at this site, as described in Section 2.1.4, and initial dose calculations using historical data support the assumption that the internal dose component of TED is expected to be the decision driver. Since the NEPTUNE detonation resulted in a small yield, some fission products are also present that may contribute to external dose.

\subsubsection{Physical Setting}

NEPTUNE is located on a south-southeast facing slope along the eastern side of the Rainier Mesa. E-Tunnel is located several hundred ft down gradient from NEPTUNE. Several small gullies are present, joining further down slope to form a larger wash. The site is at an elevation of approximately 6,600 ft above mean sea level (amsl) (Desert Research Institute [DRI], 1988).

Geologically, Rainier Mesa is comprised of a welded tuff overlying friable-bedded tuff and zeolitized-bedded tuffs of the Piapi Canyon Group and Indian Trail Formation of the Tertiary age (U.S. Geological Survey [USGS], 1965; Winograd and Thordarson, 1975). Rainier Mesa is the highest of a group of mesas, ridges, and low mountains which compose the Belted Range, and is the remnant of a volcanic plateau uplifted during an episode of tectonic extension during the middle to late Cenozoic (DRI, 1987). The tuff is up to 5,000 ft thick, and soda rhyolitic in composition. The tuff includes the Grouse Canyon Member, the most densely welded tuff; many outcrops resemble a lava rather than a welded tuff (Geological Society of America, 1968). The tuff originated from a series of calderas.

Rainier Mesa serves as part of a drainage divide that separates westerly surface drainage to the Fortymile Canyon from the easterly surface drainage to Yucca Flat (DRI, 1987). Drainage from the area is to Yucca Flat. Within the subsurface, the regional zone of saturation occurs in the Paleozoic strata several thousand $\mathrm{ft}$ beneath the surface. At Rainier Mesa, perched water occurs only within the tuff aquitard, the top of which occurs at about 6,600 ft amsl. The perched water table that exists in fractures within the aquitard occurs between 6,033 and 6,184 ft amsl in the east-central portion of Rainier. In tunnels, perched water was found in poorly connected fractures. The water table within the underlying lower carbonate aquifer exists at about 2,000 ft below the perched water table (Winograd and Thordarson, 1975). Groundwater beneath Rainier Mesa may flow westward or southward within the Alkali Flat-Furnace Creek Ranch subbasin, or some part may flow eastward (USGS, 1996).

Precipitation rates for this region average from 6 to 12 inches as indicated on isohyetal maps (USGS, 1965). Precipitation deposited on Rainier Mesa either infiltrates into soil and rock, runs off in gullies and washes, or is lost to evapotranspiration. Precipitation that infiltrates into the overlying soil and exposed rock percolates through unsaturated rock material, locally recharging the groundwater system (USGS, 1996). Recharge on top of the Mesa is estimated at 140-acre $\mathrm{ft}$ per year based on a proportional percentage of precipitation (USGS, 1996).

Well ER-12-1 is located near the base of the eastern slope of Rainier Mesa, alongside the E-Tunnel access road at the base of Dolomite Hill in Area 12, within two miles of the site. Well ER-12-1 is at 5,817 ft amsl, and was drilled to a depth of 3,588 ft in 1991. Groundwater in Well ER-12-1 was measured at approximately 1,500 ft below ground surface (bgs) (USGS and U.S. Department of Energy [DOE], 2003). 


\subsubsection{Site Access and Use}

Access to NEPTUNE is limited. The area is posted as a Contamination Area (CA) in compliance with Title 10 Code of Federal Regulations (CFR) Part 835, "Occupational Radiation Protection" (CFR, 1993). The surface of the site is steep and rocky. Access and use is limited due to the remote location, rough terrain, and steep slope of the area. Figures 5 through 7 provide historical photographs of the site. In addition, use of the area is restricted by the UR implemented during closure of CAU 551. The UR for CAU 551 covers much of the radiological plume associated with NEPTUNE as seen on the 1994 flyover survey data, and it is not possible to access the NEPTUNE crater without crossing through the CAU 551 UR.

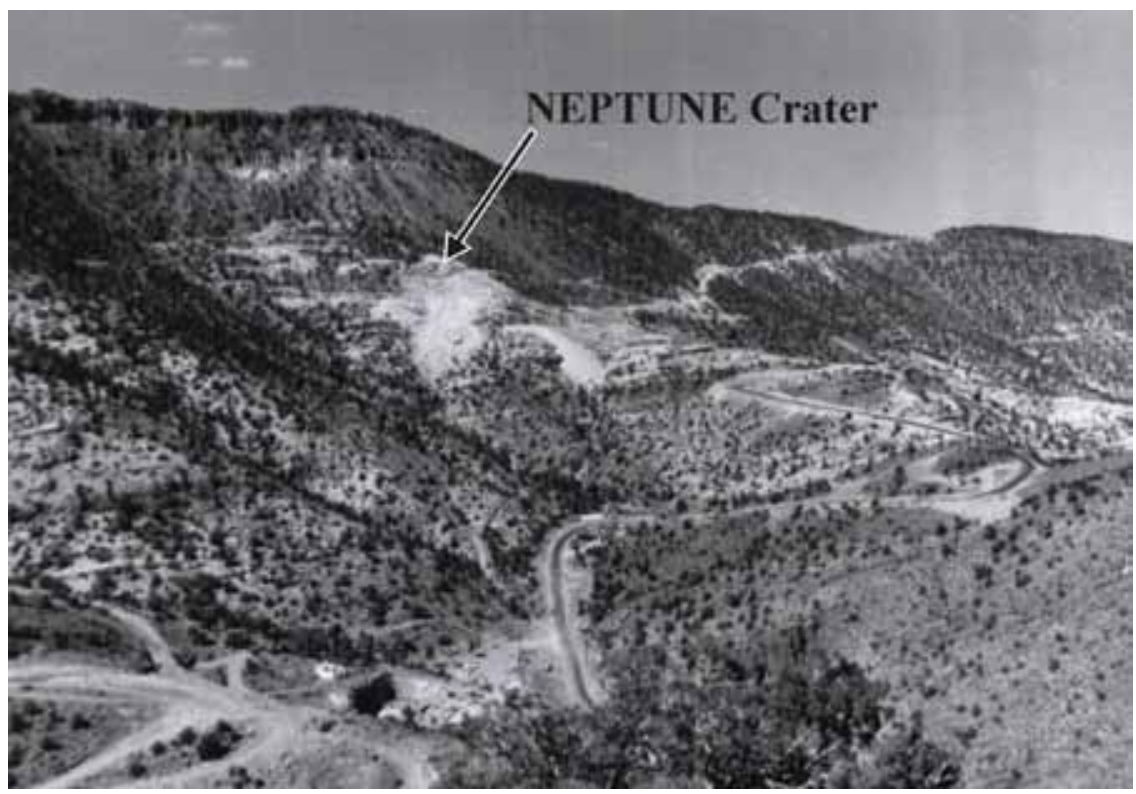

Figure 5. NEPTUNE CRATER LOCATION, 10/14/1958

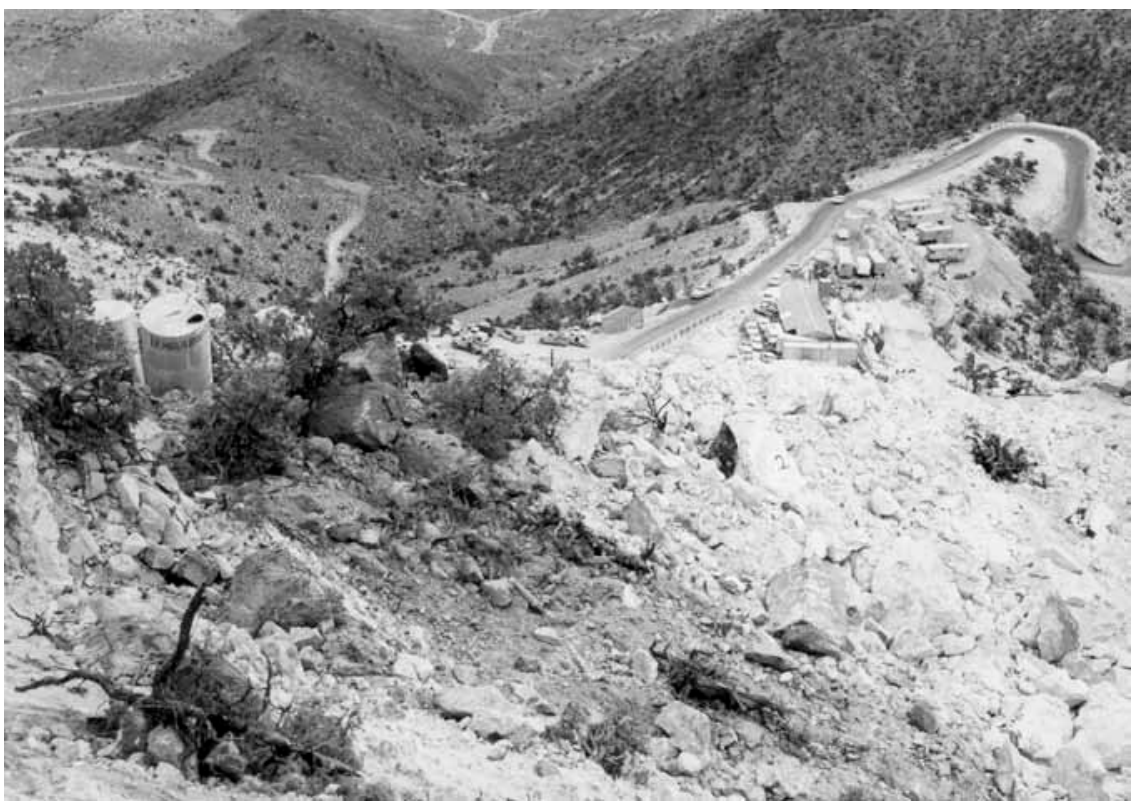

FIGURE 6. VIEW FROM RIM OF NEPTUNE CRATER, 10/23/1958 


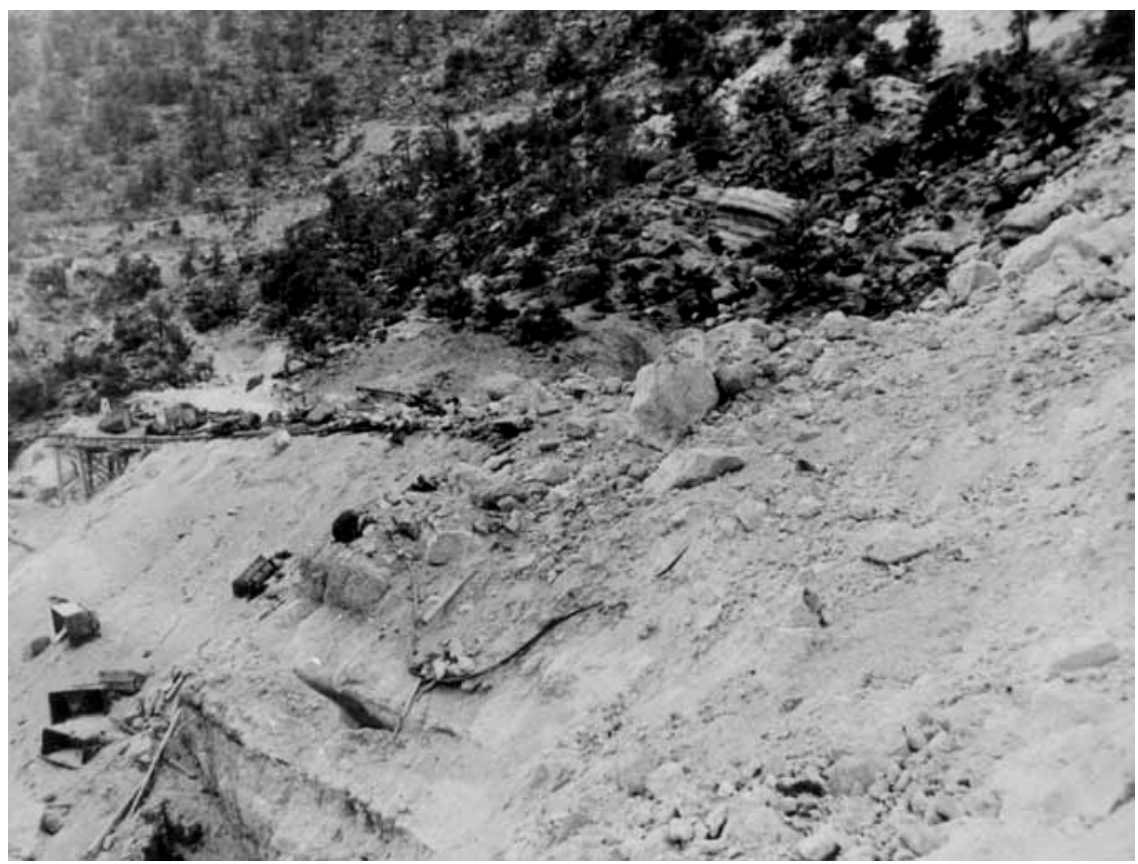

Figure 7. NEPTUNE CrATER AND Post-Shot DAMAge, 10/23/1958

\subsubsection{Available Characterization Information}

A large body of characterization information is available for NEPTUNE. These are discussed in the following sections.

\subsubsection{Aerial Radiological (Flyover) Survey Data}

In 1994, a flyover survey was conducted with 100-percent coverage of the NNSS (Hendricks and Riedhauser, 1999). Data were collected by a gamma ray detection instrument on an aircraft flown at approximately $200 \mathrm{ft}$ above ground level, with a line spacing of approximately $500 \mathrm{ft}$, and at a ground speed of approximately $127 \mathrm{ft}$ per second. Data provide an indication of whether radiological contamination is present above background levels and relative amounts of strong gamma-emitting radionuclides (fission products) and americium-241. The data show relative levels of total radiological contamination but do not provide radionuclide-specific quantities (Proctor, 1997).

Measurements for background radiation, which is radioactivity that cannot be attributed to a specific source but is due to low-level, worldwide natural and man-made sources, were continuously collected during the 1994 flyover survey. Each flyover survey measurement is corrected to remove background radiation levels, resulting in data that show the added man-made radiation resulting from testing or other activities.

The 1994 flyover survey data indicate detectable levels of americium-241 and fission products in the area of NEPTUNE (Hendricks and Riedhauser, 1999). Americium-241 implies the presence of plutonium. Americium and plutonium are the major contributors to internal dose. Fission products are the major contributors to external dose. It is anticipated that the internal dose component will be the most significant portion of TED at this site. While fission products are also present at the site, these constituents do not appear to be present at levels that would cause external dose to be the dominant dose contributor. Figure 8 shows the 1994 flyover survey data. 
CAU 574 SAFER Plan

Section: Unit Description

Revision: 0

Date: August 2011

THIS PAGE INTENTIONALLY LEFT BLANK 


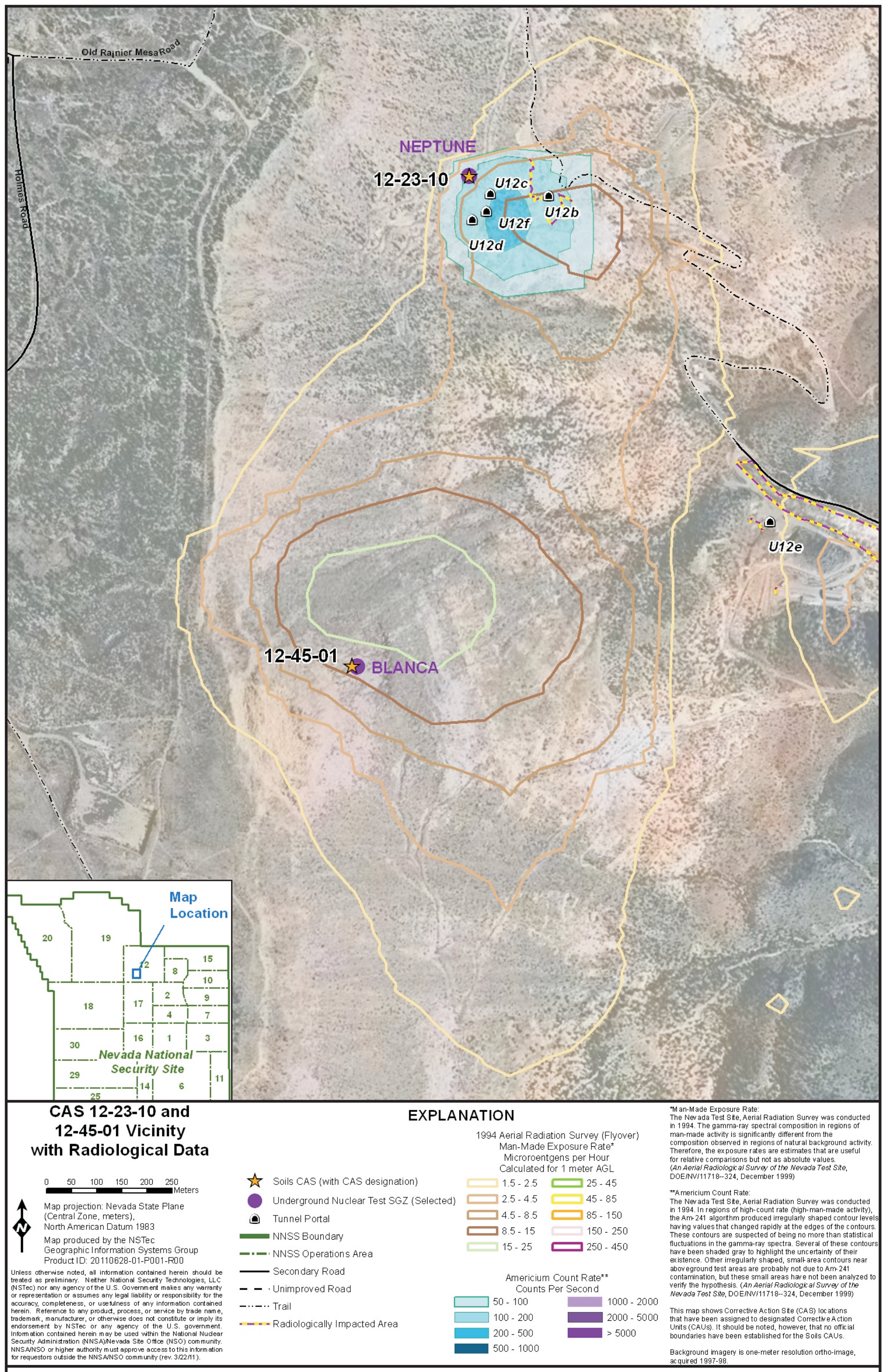

FIGURE 8

CORRECTIVE ACTION UNIT 574 FLyOVER DATA 
CAU 574 SAFER Plan

Section: Unit Description

Date: August 2011

THIS PAGE INTENTIONALLY LEFT BLANK 


\subsubsection{Corrective Action Unit 551 Characterization Data}

Analytical data collected for CAU 551 under the Industrial Sites Sub-Project will be used to support closure of NEPTUNE. The investigation of CAU 551 included the muckpiles associated with B-, C-, D-, and F-Tunnels (U12b, U12c, U12d, and U12f, respectively). As shown on Figure 9, soil samples were collected for CAU 551 within the NEPTUNE contamination plume. Radiological contamination was identified above action levels, and the CASs within CAU 551 (CASs 12-06-05, 12-06-07, and 12-06-08) were closed in place with a UR. The existing UR overlaps a large portion of the plume association with NEPTUNE. The boundary of the existing UR for CAU 551 is shown on Figure 9 (NNSA/NSO, 2006a).

A total of 77 samples were collected from 37 locations during Decision I sampling for CAU 551. A total of 87 Decision II samples were collected from 60 locations in the downslope and upslope areas from the muckpiles and along bordering roadways. Radionuclide analytical results for soil samples detected above minimum detectable concentrations that will be used for closure of NEPTUNE are presented in Table 1 (NNSA/NSO, 2006a).

The concentrations of radionuclides in subsurface soil samples collected from the muckpiles up to $3 \mathrm{ft}$ bgs support the assumption that excavated debris and muck generated during tunnel re-entry and recovery activities following the test contained radioactive material. The levels of plutonium and americium in soil sample results also confirm the assumptions that internal dose is the most significant contributor to TED at this site. The CAU 551 soil sample results will be compared to the Residual Radioactive Material Guideline (RRMGs) calculated for NEPTUNE, as discussed in Section 3.2.1, using the sum of the fractions approach to calculate internal dose rates.

\subsubsection{Radionuclide Inventory and Distribution Program Data}

In 1981 a survey of contaminated soil at the NNSS began under the Radionuclide Inventory and Distribution Program (RIDP). An in situ gamma spectroscopy technique was used to provide radionuclide-specific levels of contamination. The results were published in six reports (McArthur and Kordas, 1983 and 1985; McArthur and Mead, 1987, 1988, and 1989; McArthur, 1991). Soil samples were also collected at selected locations in increments from the surface down to 15 centimeters to determine the distribution of radionuclides with depth and the ratio of non-gamma-emitting radionuclides to gamma-emitting radionuclides.

The RIDP data can be used to estimate internal and/or external radiological dose rates using standard dose assessment models that consider the amount of contamination present and how the land will be used. While RIDP data were not collected in the immediate vicinity of NEPTUNE due to the rugged terrain and steep slope of the area, RIDP data, when averaged across associated 1994 flyover isopleths, are well correlated to 1994 flyover survey data (National Security Technologies, LLC [NSTec], 2008).

The RIDP results across the entire NNSS have been grouped according to the 1994 flyover isopleth in which they were located. The RIDP values within each group were averaged and compared to the middle value of each range. These comparisons demonstrate a linear relationship based on wide-area averages of RIDP data. Therefore, the RIDP data and 1994 flyover survey data can be used to estimate dose rates and conservatively project 25-mrem/yr boundaries for each exposure scenario. The method used to convert the RIDP data and the correlation of the RIDP data to the 1994 flyover survey data are described in Radionuclide Inventory Distribution Project Data Evaluation and Verification White Paper (NSTec, 2008). 
CAU 574 SAFER Plan

Section: Unit Description

Revision: 0

Date: August 2011

THIS PAGE INTENTIONALLY LEFT BLANK 


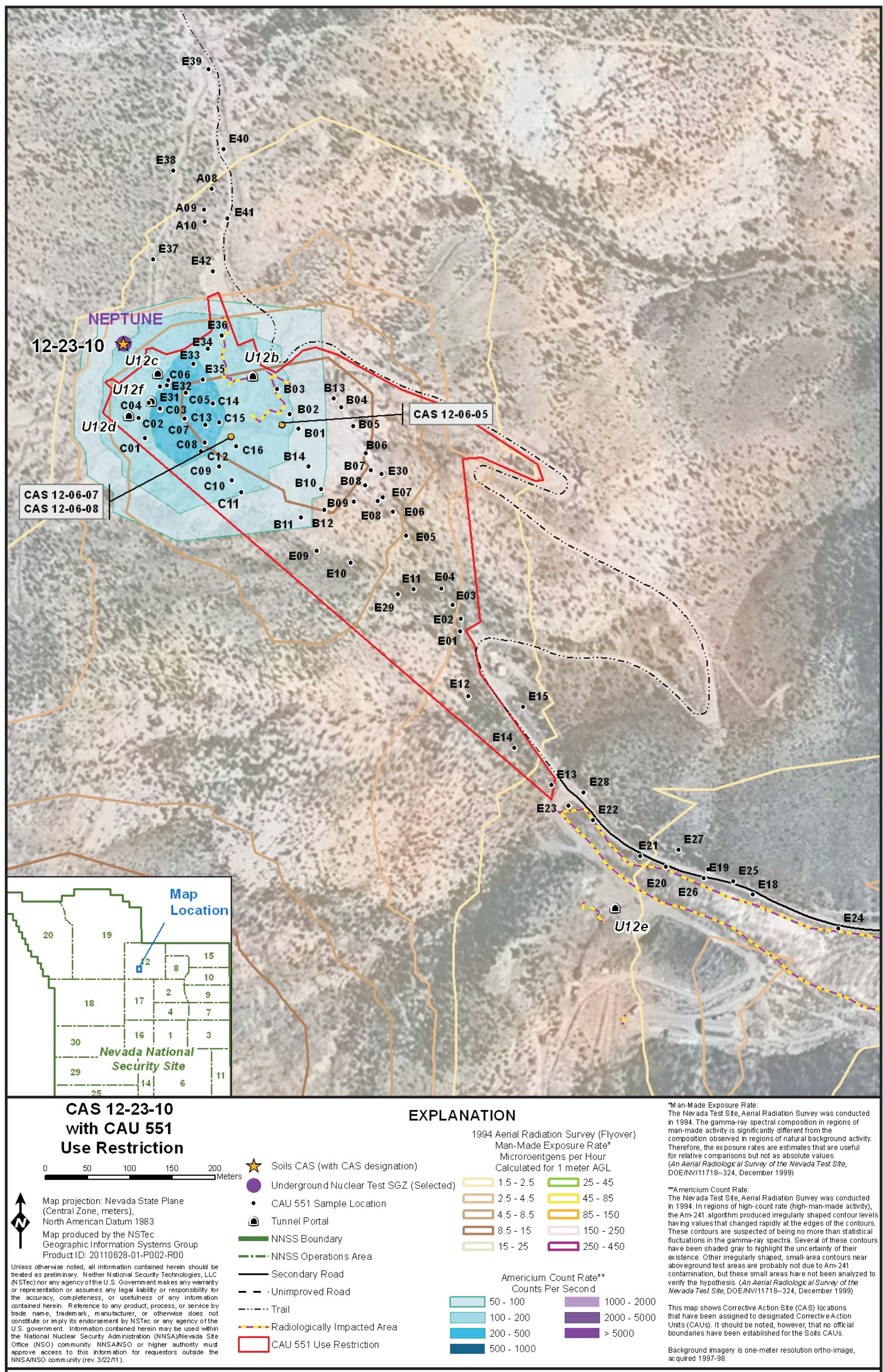

FIGURE 9

CORRECTIVE ACTION UNIT 551 SOIL SAMPLE LOCATIONS AND USE RESTRICTION BOUNDARY 
CAU 574 SAFER Plan

Section: Unit Description

Date: August 2011

THIS PAGE INTENTIONALLY LEFT BLANK 
Table 1. Corrective Action Unit 551 SoIl Sample Results

\begin{tabular}{|c|c|c|c|c|c|c|c|c|}
\hline $\begin{array}{c}\text { Sample } \\
\text { Location }\end{array}$ & $\begin{array}{l}\text { Sample } \\
\text { Number }\end{array}$ & $\begin{array}{c}\text { Depth } \\
\text { (ft bgs) }\end{array}$ & $\begin{array}{c}\text { Americium-241 } \\
\text { (pCi/g) }\end{array}$ & $\begin{array}{c}\text { Cesium-137 } \\
\text { (pCi/g) }\end{array}$ & $\begin{array}{c}\text { Europium-152 } \\
(\mathrm{pCi} / \mathrm{g})\end{array}$ & $\begin{array}{c}\text { Plutonium-238 } \\
\text { (pCi/g) }\end{array}$ & $\begin{array}{c}\text { Plutonium-239 } \\
\text { (pCi/g) }\end{array}$ & $\begin{array}{c}\text { Strontium-90 } \\
(\mathrm{pCi} / \mathrm{g})\end{array}$ \\
\hline A08 & 551A019 & $0.0-0.5$ & $6.5(\mathrm{~J})^{\mathrm{a}}$ & $5.9(\mathrm{G})$ & -- & 1.21 & 21.8 & 0.79 \\
\hline A09 & 551A020 & $0.0-0.5$ & -- & $2.32(\mathrm{G})$ & -- & 0.77 & 10.1 & -- \\
\hline A10 & 551A021 & $0.0-0.5$ & $3.38(\mathrm{~J})^{\mathrm{a}}$ & $3.28(\mathrm{G})$ & -- & 0.91 & 11.9 & -- \\
\hline \multirow{4}{*}{ B01 } & 551B001 & $0.0-0.5$ & $6.36(\mathrm{~J})^{\mathrm{a}}$ & $10(\mathrm{G})$ & -- & 0.43 & 24.6 & -- \\
\hline & 551B002 & $0.5-1.0$ & $18.6(\mathrm{~J})^{\mathrm{a}}$ & $8.2(\mathrm{G})$ & -- & 0.83 & 43.7 & 0.7 \\
\hline & 551B003 & $0.5-1.0$ & $31(\mathrm{~J})^{\mathrm{a}}$ & $8.5(\mathrm{G})$ & -- & 0.79 & 46.5 & 0.84 \\
\hline & 551B004 & $2.5-3.0$ & N/A & $1.61(\mathrm{G})$ & -- & 0.108 & 5.53 & -- \\
\hline \multirow{2}{*}{ B02 } & 551B005 & $0.0-0.5$ & $107(\mathrm{~J})^{\mathrm{b}}$ & 156(G,M3) & $26.6(\mathrm{~J})^{\mathrm{b}}$ & 26.8(M3) & 1,430(M3) & $6.3(\mathrm{~J})$ \\
\hline & 551B006 & $1.0-1.5$ & -- & $4.1(\mathrm{G})$ & -- & 0.289 & 14.4 & -- \\
\hline \multirow{2}{*}{ B03 } & 551B007 & $0.0-0.5$ & $99(J)^{b}$ & 318(G,M3) & -- & 4.07 & 222 & $11.5(\mathrm{~J})$ \\
\hline & 551B008 & $1.0-1.5$ & $55.3(\mathrm{~J})^{\mathrm{b}}$ & $103(\mathrm{G})$ & -- & 2.31(M3) & 104(M3) & 4.8 \\
\hline \multirow{2}{*}{ B04 } & 551B009 & $0.0-0.5$ & $136(\mathrm{~J})^{b}$ & $58(\mathrm{G})$ & $5.49(\mathrm{~J})^{\mathrm{b}}$ & 6.5 & 344 & 3.03 \\
\hline & 551B010 & $3.5-4.0$ & $6.67(J)^{b}$ & $14.6(\mathrm{G})$ & -- & 1 & 48 & -- \\
\hline \multirow{2}{*}{ B05 } & 551B011 & $0.0-0.5$ & $244(\mathrm{~J})^{\mathrm{b}}$ & $57.6(\mathrm{G})$ & -- & 13.5(M3) & 740(M3) & $6.3(\mathrm{~J})$ \\
\hline & 551B012 & $1.5-2.0$ & $186(\mathrm{~J})^{\mathrm{b}}$ & $33(\mathrm{G})$ & -- & 7.3 & 367(M3) & $2.55(\mathrm{~J})$ \\
\hline \multirow{2}{*}{ B06 } & 551B013 & $0.0-0.5$ & $192(\mathrm{~J})^{\mathrm{b}}$ & $38.4(\mathrm{G})$ & -- & 10.2(M3) & 555(M3) & 3.8 \\
\hline & 551B014 & $2.0-2.5$ & $144(\mathrm{~J})^{\mathrm{b}}$ & $27.5(\mathrm{G})$ & -- & 7.3(M3) & 442(M3) & 2.59 \\
\hline \multirow{2}{*}{ B07 } & 551B015 & $0.0-0.5$ & $52.5(\mathrm{~J})^{\mathrm{b}}$ & $43.9(\mathrm{G})$ & -- & 2.45(M3) & 126(M3) & $4.2(\mathrm{~J})$ \\
\hline & 551B016 & $1.0-1.5$ & $101(\mathrm{~J})^{\mathrm{b}}$ & $22.2(\mathrm{G})$ & -- & 7(M3) & 335(M3) & $1.86(\mathrm{~J})$ \\
\hline \multirow{2}{*}{ B08 } & 551B017 & $0.0-0.5$ & $19.5(\mathrm{~J})^{\mathrm{b}}$ & $22.5(\mathrm{G})$ & -- & 0.94 & 43.6 & 1.92 \\
\hline & 551B018 & $2.0-3.0$ & $33.3(\mathrm{~J})^{b}$ & $12.1(\mathrm{G})$ & -- & 1.28 & 67 & 1.22 \\
\hline \multirow{2}{*}{ B09 } & 551B019 & $0.0-0.5$ & $52.2(\mathrm{~J})^{\mathrm{b}}$ & $32.4(\mathrm{G})$ & -- & 5.1(M3) & 191(M3) & $1.49(\mathrm{~J})$ \\
\hline & 551B020 & $1.0-1.5$ & $5.7(\mathrm{~J})^{\mathrm{b}}$ & $4.09(\mathrm{G})$ & -- & 0.306 & 13.1 & -- \\
\hline \multirow{2}{*}{ B10 } & 551B021 & $0.0-0.5$ & $64.8(\mathrm{~J})^{\mathrm{b}}$ & $26.8(\mathrm{G})$ & -- & 3.34 & 171 & 2.84 \\
\hline & 551B022 & $2.0-3.0$ & $178(\mathrm{~J})^{\mathrm{b}}$ & $55.1(\mathrm{G})$ & -- & 8.6(M3) & 452(M3) & $4.8(\mathrm{~J})$ \\
\hline \multirow{2}{*}{ B11 } & 551B023 & $0.0-0.5$ & $50.5(J)^{b}$ & $22.2(\mathrm{G})$ & -- & 3.29 & 172(M3) & $3.2(\mathrm{~J})$ \\
\hline & 551B024 & $1.0-1.5$ & $14(\mathrm{~J})^{\mathrm{b}}$ & $4.66(\mathrm{G})$ & -- & 0.56 & 34.7 & 0.83 \\
\hline \multirow{3}{*}{ B12 } & 551B025 & $0.0-0.5$ & $10.4(\mathrm{~J})^{\mathrm{b}}$ & $7.4(\mathrm{G})$ & -- & 0.79 & 33 & $0.68(\mathrm{~J})$ \\
\hline & 551B026 & $0.0-0.5$ & $14(\mathrm{~J})^{\mathrm{b}}$ & $10.1(\mathrm{G})$ & -- & 0.82 & 33.6 & -- \\
\hline & 551B027 & $1.0-2.0$ & -- & $0.65(\mathrm{G})$ & -- & 0.4 & 7.8 & -- \\
\hline \multirow{2}{*}{ B13 } & 551B028 & $0.0-0.5$ & $156(\mathrm{~J})^{\mathrm{b}}$ & $39.9(\mathrm{G})$ & $1.14(\mathrm{~J})^{\mathrm{b}}$ & 6.6(M3) & 321(M3) & $3.15(\mathrm{~J})$ \\
\hline & 551B029 & $1.5-2.0$ & $10.5(\mathrm{~J})^{\mathrm{b}}$ & $5.75(\mathrm{G})$ & -- & 1.07 & 50.5 & $0.39(\mathrm{LT})$ \\
\hline B14 & 551B030 & $0.0-0.5$ & $478(\mathrm{~J})^{\mathrm{b}}$ & $164(G)$ & -- & 28.6(M3) & 1,440(M3) & 21.7 \\
\hline \multirow{3}{*}{ C01 } & 551C001 & $0.0-0.5$ & $2.66(\mathrm{~J})^{\mathrm{b}}$ & -- & -- & 0.089 & 9.7 & -- \\
\hline & $551 \mathrm{C} 002$ & $3.5-4.0$ & -- & -- & -- & -- & -- & -- \\
\hline & $551 \mathrm{C} 003$ & $3.5-4.0$ & -- & -- & -- & -- & -- & -- \\
\hline
\end{tabular}


Table 1. Corrective ACtion Unit 551 Soil SAMPle Results (CONTINUED)

\begin{tabular}{|c|c|c|c|c|c|c|c|c|}
\hline $\begin{array}{c}\text { Sample } \\
\text { Location }\end{array}$ & $\begin{array}{l}\text { Sample } \\
\text { Number }\end{array}$ & $\begin{array}{c}\text { Depth } \\
\text { (ft bgs) }\end{array}$ & $\begin{array}{c}\text { Americium-241 } \\
\text { (pCi/g) }\end{array}$ & $\begin{array}{c}\text { Cesium-137 } \\
(\mathbf{p C i} / \mathrm{g})\end{array}$ & $\begin{array}{c}\text { Europium-152 } \\
(\mathrm{pCi} / \mathrm{g})\end{array}$ & $\begin{array}{c}\text { Plutonium-238 } \\
\text { (pCi/g) }\end{array}$ & $\begin{array}{c}\text { Plutonium-239 } \\
(\mathrm{pCi} / \mathrm{g})\end{array}$ & $\begin{array}{c}\text { Strontium-90 } \\
(\mathrm{pCi} / \mathrm{g})\end{array}$ \\
\hline \multirow{2}{*}{ C02 } & $551 \mathrm{C} 004$ & $0.0-0.5$ & $172(\mathrm{~J})^{\mathrm{b}}$ & $20.4(\mathrm{G})$ & -- & 10.1(M3) & 760(M3) & $2.49(\mathrm{~J})$ \\
\hline & $551 \mathrm{C} 005$ & $1.0-1.5$ & -- & -- & -- & 0.063 & 2.64 & -- \\
\hline $\mathrm{C} 03$ & $551 \mathrm{C} 006$ & $0.0-0.5$ & $375(\mathrm{~J})^{\mathrm{b}}$ & $32.4(\mathrm{G})$ & $1.53(\mathrm{~J})^{\mathrm{b}}$ & 54.5(M3) & 4,220(M3) & $6.4(\mathrm{~J})$ \\
\hline \multirow{2}{*}{ C04 } & $551 \mathrm{C} 007$ & $0.0-0.5$ & $1,020(\mathrm{~J})^{\mathrm{b}}$ & $58.2(\mathrm{G})$ & $5.03(\mathrm{~J})^{\mathrm{b}}$ & 127(M3) & $13,200(\mathrm{M} 3)$ & $21.7(\mathrm{~J})$ \\
\hline & $551 \mathrm{C} 008$ & $1.0-1.5$ & $300(\mathrm{~J})^{\mathrm{b}}$ & $26.5(\mathrm{G})$ & $2.3(\mathrm{~J})^{\mathrm{b}}$ & 11.3(M3) & 770(M3) & $6(\mathrm{~J})$ \\
\hline \multirow{2}{*}{ C05 } & 551C009 & $0.0-0.5$ & $1,210(\mathrm{~J})^{\mathrm{b}}$ & 62.7(G,M3) & $60(\mathrm{~J})^{\mathrm{b}}$ & 53.8(M3) & 3,880(M3) & $12.7(\mathrm{~J})$ \\
\hline & 551C010 & $2.5-3.0$ & $464(\mathrm{~J})^{b}$ & $13.9(\mathrm{G})$ & $27.4(\mathrm{~J})^{\mathrm{b}}$ & 20.4(M3) & 1,560(M3) & $2.83(\mathrm{~J})$ \\
\hline \multirow{2}{*}{ C06 } & $551 \mathrm{C} 011$ & $0.0-0.5$ & $204(J)^{b}$ & $29(\mathrm{G})$ & $1.38(\mathrm{~J})^{\mathrm{b}}$ & 12.7(M3) & 810(M3) & 4.3 \\
\hline & $551 \mathrm{C} 012$ & $0.5-1.0$ & $400(\mathrm{~J})^{\mathrm{b}}$ & $25.6(\mathrm{G})$ & $2.2(\mathrm{~J})^{\mathrm{b}}$ & $8.4(\mathrm{M} 3)$ & 610(M3) & 3.19 \\
\hline \multirow{2}{*}{ C07 } & $551 \mathrm{C} 013$ & $0.0-0.5$ & $521(\mathrm{~J})^{\mathrm{b}}$ & $20.6(\mathrm{G})$ & $1.8(\mathrm{~J})^{\mathrm{b}}$ & 27.1(M3) & 1,980(M3) & 1.91 \\
\hline & $551 \mathrm{C} 014$ & $1.5-2.0$ & $253(\mathrm{~J})^{\mathrm{b}}$ & $8.2(\mathrm{G})$ & $0.75(\mathrm{~J})^{\mathrm{b}}$ & 13(M3) & 770(M3) & 0.84 \\
\hline \multirow{2}{*}{ C08 } & $551 \mathrm{C} 015$ & $0.0-0.5$ & $251(\mathrm{~J})^{b}$ & $5.02(\mathrm{G})$ & -- & 2.5 (M3) & 224(M3) & -- \\
\hline & $551 \mathrm{C} 016$ & $1.0-1.5$ & -- & -- & -- & -- & $2.43(\mathrm{~J})^{\mathrm{a}}$ & -- \\
\hline C09 & 551C017 & $0.0-0.5$ & $1,260(\mathrm{~J})^{\mathrm{b}}$ & $40.6(\mathrm{G})$ & $3.32(\mathrm{~J})^{\mathrm{b}}$ & 42.8(M3) & 3,030(M3) & 2.99 \\
\hline \multirow{2}{*}{ C10 } & 551C018 & $0.0-0.5$ & $3,370(\mathrm{~J})^{b}$ & $101(\mathrm{G})$ & $9.7(\mathrm{~J})^{\mathrm{b}}$ & $96(\mathrm{~J})^{\mathrm{d}}$ & $7,000(\mathrm{~J})^{\mathrm{d}}$ & $7.9(\mathrm{~J})$ \\
\hline & 551C019 & $1.0-1.5$ & $518(\mathrm{~J})^{\mathrm{b}}$ & $17.7(\mathrm{G})$ & $1.95(\mathrm{~J})^{\mathrm{b}}$ & 34.2(M3) & 2,530(M3) & 1.62 \\
\hline \multirow{2}{*}{ C11 } & $551 \mathrm{C} 020$ & $0.0-0.5$ & $606(\mathrm{~J})^{\mathrm{b}}$ & $27.6(\mathrm{G})$ & $1.73(\mathrm{~J})^{\mathrm{b}}$ & 18.6(M3) & 1,210(M3) & 1.59 \\
\hline & 551C021 & $2.5-3.0$ & $318(\mathrm{~J})^{b}$ & $13.5(\mathrm{G})$ & -- & 16.3(M3) & 1,230(M3) & 1.19 \\
\hline \multirow{2}{*}{ C12 } & $551 \mathrm{C} 022$ & $0.0-0.5$ & $500(J)^{b}$ & $22.5(\mathrm{G})$ & $1.78(\mathrm{~J})^{\mathrm{b}}$ & 26.6(M3) & 1,850(M3) & $2.05(\mathrm{~J})$ \\
\hline & $551 \mathrm{C} 023$ & $1.0-1.5$ & $701(\mathrm{~J})^{\mathrm{b}}$ & $32.1(\mathrm{G})$ & $1.93(\mathrm{~J})^{\mathrm{b}}$ & 25.6(M3) & 1,820(M3) & $2.39(\mathrm{~J})$ \\
\hline \multirow{2}{*}{ C13 } & $551 \mathrm{C} 024$ & $0.0-0.5$ & $519(\mathrm{~J})^{b}$ & $16.2(\mathrm{G})$ & $29.3(\mathrm{~J})^{b}$ & 30.1(M3) & 2,280 (M3) & $3.51(\mathrm{~J})$ \\
\hline & $551 \mathrm{C} 025$ & $2.0-3.0$ & $515(\mathrm{~J})^{\mathrm{b}}$ & $35.4(\mathrm{G})$ & $11.7(\mathrm{~J})^{\mathrm{b}}$ & 18.9(M3) & 1,390(M3) & 3.9(J,Y1) \\
\hline \multirow{3}{*}{ C14 } & 551C026 & $0.0-0.5$ & $489(\mathrm{~J})^{b}$ & 19.9(G) & $14.8(\mathrm{~J})^{b}$ & 21.7(M3) & 1,570(M3) & $3.8(\mathrm{~J})$ \\
\hline & $551 \mathrm{C} 027$ & $0.0-0.5$ & $415(\mathrm{~J})^{\mathrm{b}}$ & 19.1(G) & $14.8(\mathrm{~J})^{\mathrm{b}}$ & 26.6(M3) & $1,840(\mathrm{M} 3)$ & 4.3(J,Y1) \\
\hline & $551 \mathrm{C} 028$ & $1.0-1.5$ & $202(\mathrm{~J})^{\mathrm{b}}$ & $12.8(\mathrm{G})$ & $6.9(\mathrm{~J})^{\mathrm{b}}$ & 13.8(M3) & 1,050(M3) & $3.71(\mathrm{~J}, \mathrm{Y} 1)$ \\
\hline \multirow{2}{*}{ C15 } & 551C029 & $0.0-0.5$ & $159(\mathrm{~J})^{\mathrm{b}}$ & $19.8(\mathrm{G})$ & -- & 10(M3) & 730(M3) & $3.48(\mathrm{Y} 1)$ \\
\hline & $551 \mathrm{C} 030$ & $1.0-1.5$ & $264(\mathrm{~J})^{b}$ & $25.9(\mathrm{G})$ & $1.97(\mathrm{~J})^{\mathrm{b}}$ & 16.1(M3) & 1,200 (M3) & $4.4(Y 1)$ \\
\hline \multirow{2}{*}{ C16 } & 551C031 & $0.0-0.5$ & $34.2(\mathrm{~J})^{\mathrm{b}}$ & $8.5(\mathrm{G})$ & $1.23(\mathrm{~J})^{\mathrm{b}}$ & 1.9 & 133 & $2.47(\mathrm{Y} 1)$ \\
\hline & $551 \mathrm{C} 032$ & $2.0-2.5$ & -- & $11.1(\mathrm{G})$ & -- & 0.11 & 5.8 & 3.51(Y1) \\
\hline \multirow{2}{*}{ E01 } & 551E001 & $0.0-0.5$ & $33(J)^{\mathrm{C}}$ & $9.3(\mathrm{~J})^{\mathrm{a}}$ & -- & $1.04(\mathrm{~J})^{\mathrm{a}}$ & 44 & -- \\
\hline & 551E002 & $1.0-1.5$ & $1.8(\mathrm{~J})^{\mathrm{C}}$ & $1.23(\mathrm{~J})^{\mathrm{a}}$ & -- & $0.39(\mathrm{~J})^{\mathrm{a}}$ & 19.5 & -- \\
\hline \multirow{3}{*}{ E02 } & 551E003 & $0.0-0.5$ & $1.02(J)^{\mathrm{C}}$ & $2.06(\mathrm{~J})^{\mathrm{a}}$ & -- & $0.57(\mathrm{~J})^{\mathrm{a}}$ & 7.6 & -- \\
\hline & 551E004 & $0.0-0.5$ & $1.2(\mathrm{~J})^{\mathrm{C}}$ & $1.92(\mathrm{~J})^{\mathrm{a}}$ & -- & $0.52(\mathrm{~J})^{\mathrm{a}}$ & 7.9(M3) & -- \\
\hline & 551E005 & $1.0-1.5$ & $0.65(J)^{\mathrm{C}}$ & $2.39(\mathrm{~J})^{\mathrm{a}}$ & -- & $0.153(\mathrm{~J})^{\mathrm{a}}$ & 4.76 & -- \\
\hline E03 & 551E006 & $0.0-0.5$ & $71.5(\mathrm{~J})^{\mathrm{C}}$ & $31.7(\mathrm{~J})^{\mathrm{a}}$ & $1.51(\mathrm{~J}) \mathrm{C}$ & $3.78(\mathrm{~J})^{\mathrm{a}}$ & 195(Y1) & $1.51(\mathrm{Y} 1)$ \\
\hline E04 & 551E007 & $0.0-0.5$ & $87(\mathrm{~J})^{\mathrm{C}}$ & $26.9(\mathrm{~J})^{\mathrm{a}}$ & -- & $4.15(\mathrm{~J})^{\mathrm{a}}$ & $221(\mathrm{~J})^{\mathrm{d}}$ & 1.66 \\
\hline
\end{tabular}


Table 1. Corrective ACtion Unit 551 Soil SAMPle Results (CONTINUED)

\begin{tabular}{|c|c|c|c|c|c|c|c|c|}
\hline $\begin{array}{c}\text { Sample } \\
\text { Location }\end{array}$ & $\begin{array}{l}\text { Sample } \\
\text { Number }\end{array}$ & $\begin{array}{c}\text { Depth } \\
\text { (ft bgs) }\end{array}$ & $\begin{array}{c}\text { Americium-241 } \\
\text { (pCi/g) }\end{array}$ & $\begin{array}{c}\text { Cesium-137 } \\
\text { (pCi/g) }\end{array}$ & $\begin{array}{c}\text { Europium-152 } \\
(\mathrm{pCi} / \mathrm{g})\end{array}$ & $\begin{array}{c}\text { Plutonium-238 } \\
\text { (pCi/g) }\end{array}$ & $\begin{array}{c}\text { Plutonium-239 } \\
(\mathrm{pCi} / \mathrm{g})\end{array}$ & $\begin{array}{c}\text { Strontium-90 } \\
(\mathrm{pCi} / \mathrm{g})\end{array}$ \\
\hline & 551E008 & $1.0-1.5$ & $16.6(\mathrm{~J})^{\mathrm{C}}$ & $27.8(\mathrm{~J})^{\mathrm{a}}$ & -- & $1.95(\mathrm{~J})^{\mathrm{a}}$ & 63 & $1.17(\mathrm{Y} 1)$ \\
\hline E05 & 551E009 & $0.0-0.5$ & $94(\mathrm{~J})^{\mathrm{b}}$ & $19.9(\mathrm{G})$ & -- & $2.69(\mathrm{Y} 2)$ & 132(Y2) & 0.93 \\
\hline \multirow{2}{*}{ E06 } & 551E010 & $0.0-0.5$ & $137(\mathrm{~J})^{\mathrm{b}}$ & $23.1(\mathrm{G})$ & -- & 4.65 & 240 & 1.79 \\
\hline & 551E011 & $1.0-1.5$ & $131(\mathrm{~J})^{b}$ & $35(\mathrm{G})$ & -- & 6(M3) & 309(M3) & $2.05(\mathrm{Y} 2)$ \\
\hline E07 & 551E012 & $0.0-0.5$ & $198(\mathrm{~J})^{\mathrm{b}}$ & $27.4(\mathrm{G})$ & -- & 4.93 & 247 & $1.22(\mathrm{Y} 1)$ \\
\hline \multirow{2}{*}{ E08 } & 551E013 & $0.0-0.5$ & -- & $0.77(\mathrm{G})$ & -- & -- & 2.47 & -- \\
\hline & 551E014 & $1.0-1.5$ & -- & -- & -- & -- & 0.7 & -- \\
\hline \multirow{2}{*}{ E09 } & 551E015 & $0.0-0.5$ & $18.2(\mathrm{~J})^{\mathrm{C}}$ & $9.7(\mathrm{~J})^{\mathrm{a}}$ & -- & $2.03(\mathrm{~J})^{\mathrm{a}}$ & 82 & 2.98 \\
\hline & 551E016 & $1.5-2.0$ & $3.9(\mathrm{~J})^{\mathrm{C}}$ & $2.18(\mathrm{~J})^{\mathrm{a}}$ & -- & $0.192(\mathrm{~J})^{\mathrm{a}}$ & 13.8 & -- \\
\hline \multirow{2}{*}{ E10 } & 551E017 & $0.0-0.5$ & $487(J)^{\mathrm{C}}$ & $24.6(\mathrm{~J})^{\mathrm{a}}$ & $1.74(\mathrm{~J})^{\mathrm{C}}$ & $4.2(\mathrm{~J})^{\mathrm{a}}$ & 329(M3) & 3.54 \\
\hline & 551E018 & $1.0-1.5$ & $3.9(\mathrm{~J})^{\mathrm{C}}$ & $2.57(\mathrm{~J})^{\mathrm{a}}$ & -- & $0.95(\mathrm{~J})^{\mathrm{a}}$ & 18.4 & -- \\
\hline \multirow{2}{*}{ E11 } & 551E019 & $0.0-0.5$ & $5.9(\mathrm{~J})^{\mathrm{C}}$ & $7.23(\mathrm{~J})^{\mathrm{a}}$ & -- & $2.24(\mathrm{~J})^{\mathrm{a}}$ & 35.6 & $0.71(\mathrm{Y} 1)$ \\
\hline & 551E020 & $1.0-1.5$ & $0.09(\mathrm{~J})^{\mathrm{C}}$ & $0.17(\mathrm{~J})^{\mathrm{a}}$ & -- & $0.106(\mathrm{~J})^{\mathrm{a}}$ & 0.65 & -- \\
\hline E12 & 551E021 & $0.0-0.5$ & $-1.3(\mathrm{~J})^{\mathrm{C}}$ & $1.99(\mathrm{~J})^{\mathrm{a}}$ & -- & $0.36(\mathrm{~J})^{\mathrm{a}}$ & 2.2 & -- \\
\hline E13 & 551E022 & $0.0-0.5$ & $59.1(\mathrm{~J})^{\mathrm{C}}$ & $23.2(\mathrm{~J})^{\mathrm{a}}$ & -- & $2.6(\mathrm{~J})^{\mathrm{a}}$ & 132 & $1.24(\mathrm{Y} 1)$ \\
\hline \multirow{2}{*}{ E14 } & 551E023 & $0.0-0.5$ & $92(\mathrm{~J})^{\mathrm{C}}$ & $48.7(\mathrm{~J})^{\mathrm{a}}$ & $1.48(\mathrm{~J})^{\mathrm{C}}$ & $3.22(\mathrm{~J})^{\mathrm{a}}$ & 166(M3) & 3.07(Y1) \\
\hline & 551E024 & $0.0-0.5$ & $77.6(\mathrm{~J})^{\mathrm{C}}$ & $48.6(J)^{\mathrm{a}}$ & $1.59(\mathrm{~J})^{\mathrm{C}}$ & $4.57(\mathrm{~J})^{\mathrm{a}}$ & 229(M3) & 2.5 \\
\hline E15 & 551E025 & $0.0-0.5$ & $0.2(\mathrm{~J})^{\mathrm{C}}$ & $0.34(\mathrm{~J})^{\mathrm{a}}$ & -- & $0.082(\mathrm{~J})^{\mathrm{a}}$ & 0.43 & -- \\
\hline E18 & 551E028 & $0.0-0.5$ & -- & $0.51(\mathrm{G})$ & -- & 0.099 & 0.27 & -- \\
\hline E19 & 551E029 & $0.0-0.5$ & -- & $1.05(\mathrm{G})$ & -- & 0.149 & 0.395 & -- \\
\hline \multirow{2}{*}{ E20 } & 551E030 & $0.0-0.5$ & -- & -- & -- & 0.124 & 0.231 & -- \\
\hline & 551E031 & $0.5-1.0$ & -- & -- & -- & -- & 0.67 & -- \\
\hline E21 & 551E032 & $0.0-0.5$ & -- & -- & -- & -- & $0.035(\mathrm{LT})$ & -- \\
\hline \multirow{2}{*}{ E22 } & 551E033 & $0.0-0.5$ & -- & $0.32(\mathrm{G})$ & -- & 0.83 & 2.16 & -- \\
\hline & 551E034 & $0.5-1.0$ & -- & N/A & -- & -- & 0.39 & -- \\
\hline \multirow{2}{*}{ E23 } & 551E035 & $0.0-0.5$ & -- & $1.7(\mathrm{G})$ & -- & -- & 0.136 & -- \\
\hline & 551E036 & $0.5-1.0$ & -- & $0.85(\mathrm{G})$ & -- & 0.076 & 0.396 & -- \\
\hline \multirow{2}{*}{ E24 } & 551E037 & $0.0-0.5$ & -- & N/A & -- & 0.277 & 1.15 & -- \\
\hline & 551E038 & $0.5-1.0$ & -- & -- & -- & 0.139 & 0.56 & -- \\
\hline E25 & 551E039 & $0.0-0.5$ & -- & $0.77(\mathrm{G})$ & -- & 0.52 & 14.9 & -- \\
\hline \multirow{3}{*}{ E26 } & 551E040 & $0.0-0.5$ & -- & $0.81(\mathrm{G})$ & -- & -- & 0.86 & -- \\
\hline & 551E041 & $0.0-0.5$ & -- & $0.97(\mathrm{G})$ & -- & $0.41(\mathrm{~J})^{\mathrm{a}}$ & $3.15(\mathrm{~J})^{\mathrm{a}}$ & -- \\
\hline & 551E042 & $0.5-1.0$ & -- & $1.38(\mathrm{G})$ & -- & $0.074(\mathrm{~J})^{\mathrm{a}}$ & $1.86(\mathrm{~J})^{\mathrm{a}}$ & -- \\
\hline E27 & 551E043 & $0.0-0.5$ & -- & $1.38(\mathrm{G})$ & -- & $0.112(\mathrm{~J})^{\mathrm{a}}$ & $0.98(\mathrm{~J})^{\mathrm{a}}$ & -- \\
\hline \multirow{2}{*}{ E28 } & 551E044 & $0.0-0.5$ & -- & $1.18(\mathrm{G})$ & -- & $0.34(\mathrm{~J})^{\mathrm{a}}$ & $2.89(\mathrm{~J})^{\mathrm{a}}$ & -- \\
\hline & 551E045 & $0.5-1.0$ & -- & $1.12(\mathrm{G})$ & -- & $0.005(\mathrm{~J})^{\mathrm{a}}$ & $-0.001(\mathrm{~J})^{\mathrm{a}}$ & -- \\
\hline
\end{tabular}


Table 1. Corrective ACtion Unit 551 Soil SAMPle Results (CONTINUED)

\begin{tabular}{|c|c|c|c|c|c|c|c|c|}
\hline $\begin{array}{l}\text { Sample } \\
\text { Location }\end{array}$ & $\begin{array}{l}\text { Sample } \\
\text { Number }\end{array}$ & $\begin{array}{l}\text { Depth } \\
\text { (ft bgs) }\end{array}$ & $\begin{array}{c}\text { Americium-241 } \\
\text { (pCi/g) }\end{array}$ & $\begin{array}{l}\text { Cesium-137 } \\
\text { (pCi/g) }\end{array}$ & $\begin{array}{c}\text { Europium-152 } \\
\text { (pCi/g) }\end{array}$ & $\begin{array}{l}\text { Plutonium-238 } \\
\text { (pCi/g) }\end{array}$ & $\begin{array}{c}\text { Plutonium-239 } \\
\text { (pCi/g) }\end{array}$ & $\begin{array}{l}\text { Strontium-90 } \\
\text { (pCi/g) }\end{array}$ \\
\hline \multirow{2}{*}{ E29 } & 551E046 & $0.0-0.5$ & $0.96(\mathrm{~J})^{\mathrm{C}}$ & $0.74(\mathrm{G})$ & --- & $5.8(\mathrm{M} 3)$ & 318(M3) & $1.95(\mathrm{Y} 1)$ \\
\hline & 551E047 & $1.0-1.5$ & -- & -- & -- & $0.158(\mathrm{~J})^{\mathrm{a}}$ & $6.4(\mathrm{~J})^{\mathrm{a}}$ & -- \\
\hline \multirow{2}{*}{ E30 } & 551E048 & $0.0-0.5$ & $101(\mathrm{~J})^{\mathrm{C}}$ & $36.6(\mathrm{G})$ & -- & $5.8(\mathrm{M} 3)$ & 318(M3) & $1.95(\mathrm{Y} 1)$ \\
\hline & 551E049 & $2.0-2.5$ & -- & $0.99(\mathrm{G})$ & -- & $0.158(\mathrm{~J})^{\mathrm{a}}$ & $6.4(\mathrm{~J})^{\mathrm{a}}$ & -- \\
\hline \multirow{2}{*}{ E31 } & 551E052 & $0.0-0.5$ & $319(\mathrm{~J})^{\mathrm{a}}$ & 43.4(G,M3) & -- & $26.1(\mathrm{~J})$ & 2,080(M3) & 6.9 \\
\hline & 551E053 & $0.5-1.0$ & $347(\mathrm{~J})^{\mathrm{a}}$ & $55.2(\mathrm{G})$ & -- & $18.9(\mathrm{~J})$ & 1,140(M3) & 9.7 \\
\hline E32 & 551E054 & $0.0-0.5$ & $395(\mathrm{~J})^{\mathrm{a}}$ & $29.7(\mathrm{G})$ & $4.93(\mathrm{~J})^{\mathrm{a}}$ & $18.9(\mathrm{~J})$ & 1,060(M3) & 5.5 \\
\hline E33 & 551E055 & $0.0-0.5$ & $90(\mathrm{~J})^{\mathrm{a}}$ & $5.44(\mathrm{G})$ & -- & 2.68 & 183 & 1.21 \\
\hline \multirow{2}{*}{ E34 } & 551E056 & $0.0-0.5$ & $389(\mathrm{~J})^{\mathrm{a}}$ & $24.5(\mathrm{G})$ & $2.06(\mathrm{~J})^{\mathrm{a}}$ & 29.3(M3) & $2,230(\mathrm{M} 3)$ & 2.48 \\
\hline & 551E057 & $0.5-1.0$ & $263(\mathrm{~J})^{\mathrm{a}}$ & $17.5(\mathrm{G})$ & $1.58(\mathrm{~J})^{\mathrm{a}}$ & 12.7(M3) & 820(M3) & 1.87 \\
\hline E35 & 551E058 & $0.0-0.5$ & $135(\mathrm{~J})^{\mathrm{a}}$ & $9(\mathrm{G})$ & $1.23(\mathrm{~J})^{\mathrm{a}}$ & $3.1(\mathrm{~J})$ & 213(M3) & 2.08 \\
\hline \multirow{2}{*}{ E36 } & 551E059 & $0.0-0.5$ & $7.05(\mathrm{~J})^{\mathrm{a}}$ & $1.18(\mathrm{G})$ & -- & $1.15(\mathrm{~J})$ & 72(M3) & -- \\
\hline & 551E060 & $0.5-1.0$ & $2.16(\mathrm{~J})^{\mathrm{a}}$ & $0.57(\mathrm{G})$ & -- & $0.119(\mathrm{~J})$ & 7.3 & -- \\
\hline \multirow{2}{*}{ E37 } & 551E061 & $0.0-0.5$ & -- & -- & -- & -- & 0.89 & -- \\
\hline & 551E062 & $0.5-1.0$ & -- & -- & -- & -- & 0.157 & -- \\
\hline E38 & 551E063 & $0.0-0.5$ & $0.78(\mathrm{~J})^{\mathrm{a}}$ & $1.07(\mathrm{G})$ & -- & 0.36 & 4.53 & -- \\
\hline E39 & 551E064 & $0.0-0.5$ & -- & $0.47(\mathrm{G}, \mathrm{LT})$ & -- & 0.133 & 1.62 & -- \\
\hline \multirow{2}{*}{ E40 } & 551E065 & $0.0-0.5$ & -- & -- & -- & -- & 0.41 & -- \\
\hline & 551E066 & $0.5-1.0$ & -- & -- & -- & -- & -- & -- \\
\hline E41 & 551E067 & $0.0-0.5$ & -- & $0.51(\mathrm{G})$ & -- & 0.099 & 1.62 & -- \\
\hline \multirow{3}{*}{ E42 } & 551E068 & $0.0-0.5$ & $0.92(\mathrm{~J})^{\mathrm{a}}$ & $0.66(\mathrm{G})$ & -- & 0.58 & 6.5 & -- \\
\hline & 551E069 & $0.0-0.5$ & $0.66(\mathrm{~J})^{\mathrm{a}}$ & $0.75(\mathrm{G})$ & -- & 0.357 & 3.23 & -- \\
\hline & 551E070 & $0.5-1.0$ & -- & -- & -- & -- & 2.12 & -- \\
\hline
\end{tabular}

${ }^{\text {a }}$ Duplicate precision analysis (relative percent difference) outside control limits.

${ }^{\mathrm{b}}$ Sample does not meet counting geometry requirements.

${ }^{c}$ Duplicate normalized difference outside control limits.

${ }^{\mathrm{d}}$ Chemical yield above control limits.

$\mathrm{ft}$ bgs $=$ foot (feet) below ground surface

$\mathrm{G}=$ Sample density differs by more than 15 percent of laboratory control sample density.

$\mathrm{J}=$ estimated value

LT = Result less than requested MDC, but greater than sample-specific MDC.

M3 = Requested MDC was not met, but reported activity is greater than MDC.

Ci $/ g$ = picocurie $(\mathrm{s})$ per gram

Y1 = Chemical yield is in control at 100-110 percent. Quantitative yield is assumed.

$\mathrm{MDC}=$ minimum detectable concentration

-- = not detected above minimum reporting limits. 


\subsection{CORRECTIVE ACtion Site 12-45-01, U12E.05 Crater (BlanCA)}

CAS 12-45-01 (referred to as BLANCA in this document) consists of soil contamination associated with the BLANCA underground nuclear detonation.

\subsubsection{History and Process Knowledge}

The BLANCA detonation, conducted on October 30, 1958, was the largest and last test in Operation Hardtack II. Sponsored by LLNL, the test was a weapons-related experiment detonated in E-Tunnel, $988 \mathrm{ft}$ below the surface, and had a yield of approximately 22 kilotons (DOE/NV, 2000). BLANCA was detonated beneath the very steep face of the mesa and vented out the face of the mesa. Cap rock and boulders were loosened from the slope of the mesa, and a large section of the mesa edge shifted down slope and formed a scarp $70 \mathrm{ft}$ long. A plume of steam and dust rose 1,000 ft in the air (Johnson and Violet, 1958). A photograph of the BLANCA detonation is provided in Figure 10.

Steam at a temperature of approximately 1,000 degrees Fahrenheit was released to the sloped face of the mesa, killing the vegetation and sterilizing the soil (Ristvet, 2011). The fraction of radioactivity that was released is estimated at 0.1 percent (Johnson and Violet, 1958). The BLANCA area requires no radiological controls for compliance with 10 CFR 835 (CFR, 1993); therefore, the site is not posted for radiological concerns.

BLANCA was a standard weapons-related experiment in which a fission event occurred. The high yield of BLANCA implies that much of the original weapon was consumed and less material was available for dispersion to the environment. Since BLANCA had a fission yield, the primary contaminants present at the surface are expected to be fission products. Fission products include high-level gamma emissions that lead to external dose. Therefore, the external dose component of TED is expected to be the decision driver at this site. This assumption is supported by the 1994 flyover survey data, in which only fission products were detected.

It is assumed that subsurface contamination is present in the location where the test vented. In the area surrounding the vent, contamination is assumed to be present only in the surface soil (to a depth of 5 centimeters [cm]). Numerous studies of soils contaminated by atmospheric deposition following nuclear testing at have shown that some 90 percent of the radioactivity in undisturbed soil is contained within the top $5 \mathrm{~cm}$ of soil (McArthur and Kordas, 1983 and 1985; Gilbert et al., 1977; Tamura, 1977). The studies show that as radiological contaminants migrate downward in soils with water infiltration, the contaminant concentration profile exhibits a lognormal distribution with the maximum value remaining near the surface.

\subsubsection{Physical Setting}

BLANCA is located approximately 3,700 ft south-southwest of NEPTUNE on an east-facing slope along the eastern side of the Rainier Mesa. Information on the topography, geology, and meteorology of the site is found in Section 2.1.2.

\subsubsection{Site Access and Use}

Access to BLANCA is limited due to its remote location and very steep terrain. There is no anticipated future use of this area, and the steep slope and rough terrain surrounding the site essentially precludes access and use. Figure 11 provides a recent photograph of the site, and Figure 12 is a Google Earth image of the terrain surrounding the area. 


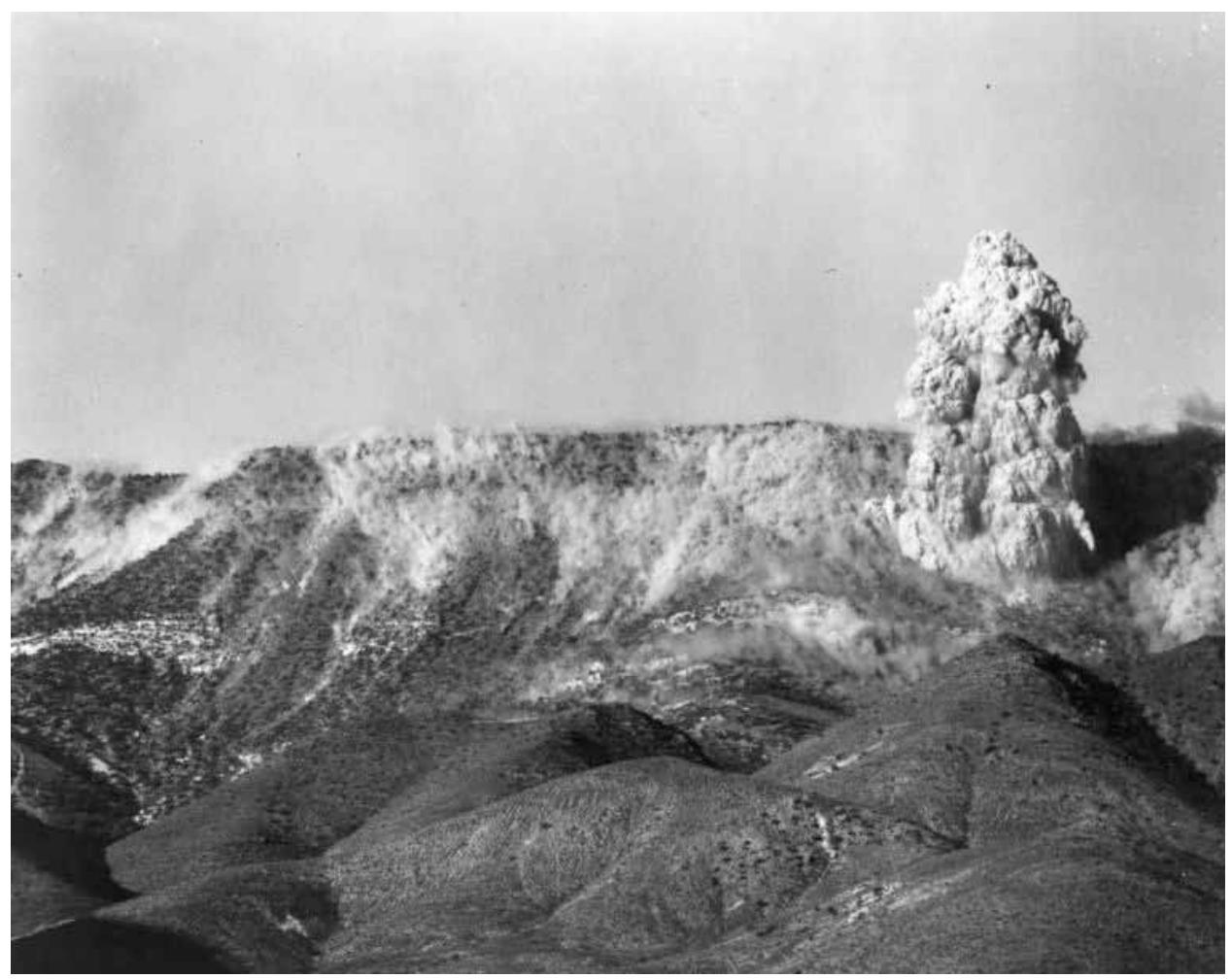

Figure 10. BLANCA DETONATION, 10/30/1958

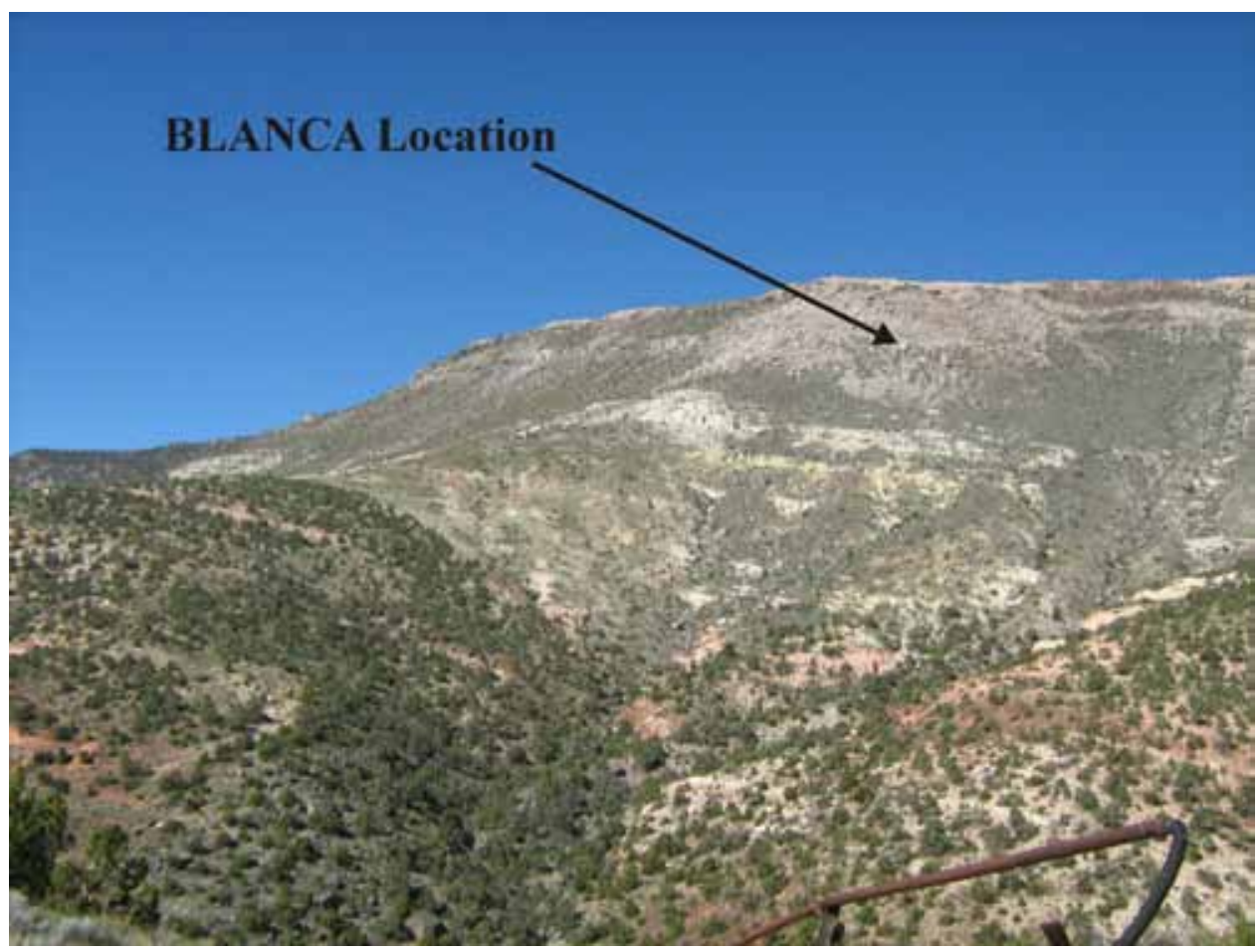

FIGURE 11. BLANCA LOCATION, 05/12/2011 


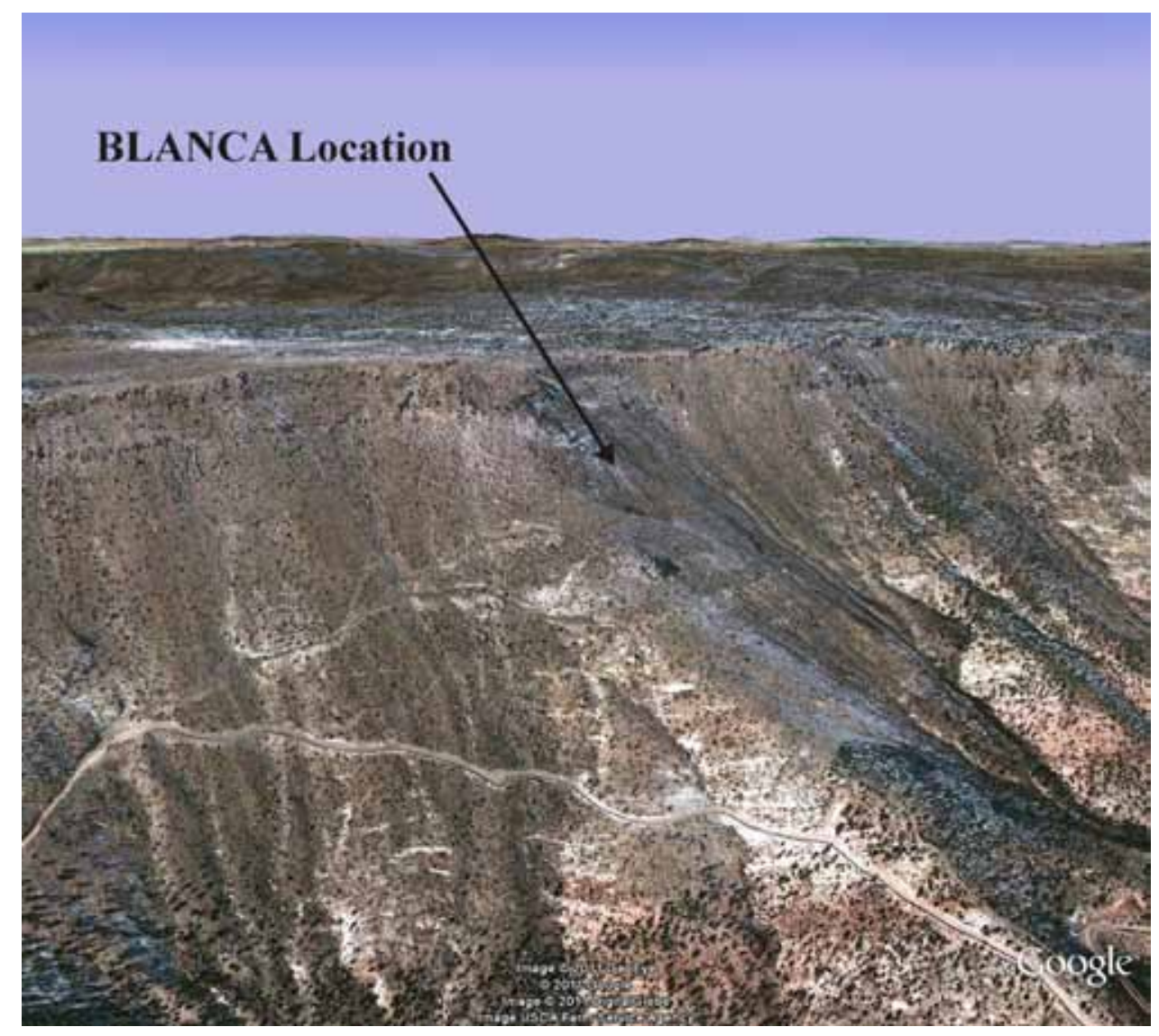

Figure 12. Google EARTH TERRAIN VIEW OF BLANCA AREA

\subsubsection{Available Characterization Information}

The sources of characterization information available for BLANCA are discussed in the following sections.

\subsubsection{Aerial Radiological (Flyover) Survey Data}

The 1994 flyover survey data indicate detectable levels of fission products in the area of BLANCA (Hendricks and Riedhauser, 1999). Americium-241 was not detected in the area of BLANCA. Americium-241 and associated plutonium are the primary internal dose drivers. Since these radionuclides were not detected, it can be assumed that the internal dose component does not significantly contribute to the TED at this site. Figure 8 shows the 1994 flyover survey data. Section 2.1.4.1 contains additional information on the 1994 flyover survey.

\subsubsection{Radionuclide Inventory and Distribution Program Data}

RIDP data were not collected in the immediate vicinity of BLANCA due to the rugged terrain and steep slope of the area. However, the RIDP data correlated to 1994 flyover survey data can be used to estimate dose rates and conservatively project 25-mrem/yr boundaries for each exposure scenario (NSTec, 2008). Section 2.1.4.3 contains additional information on the RIDP data. 
CAU 574 SAFER Plan

Section: Unit Description

Revision: 0

Date: August 2011

THIS PAGE INTENTIONALLY LEFT BLANK 


\subsection{DATA QUALITY OBJECTIVES}

The DQO process is a strategic planning approach based on the scientific method that is designed to ensure that the data collected will provide sufficient and reliable information to identify, evaluate, and technically defend the recommendation of viable corrective actions (U.S. Environmental Protection Agency [EPA], 2006). This section contains a summary of the DQO process that was developed at a meeting with decision makers on May 23, 2011.

\subsection{SUMMARY OF DQO ANALYSIS}

The DQOs are designed to identify data needs, clearly define the intended use of the data, and to design a data collection program that will satisfy these purposes. During the DQO discussions for this CAU, the informational inputs and data needs to resolve the problem statement and decisions were identified and documented.

The problem statement for CAU 574 is: "Existing information is insufficient to determine the extent of contamination and confirm closure of CAU 574.” To address this question, the resolution of two decisions statements is required:

- Decision I: "Is radiological contamination present at concentrations such that the TED rate exceeds the FAL?"

- Decision II: “If radiological contamination is present at concentrations such that the TED rate exceeds the FAL, is sufficient information available to define the extent of contamination that exceeds the FAL?"

If sufficient information is not available to meet the closure objectives, then site conditions will be re-evaluated and additional data will be collected (as long as the scope of the CAI is not exceeded and CSM assumptions have not been shown to be incorrect).

Previous data that have been collected, including analytical soil sample data, aerial radiological survey results, RIDP data, and dose rate measurements, will be evaluated to resolve the decision statements. In addition, new data will be collected to verify the adequacy of existing information, affirm the chosen corrective action, provide sufficient data to implement the corrective action, and confirm that closure objectives were met. Data that will be collected during the SAFER process to support closure of CAU 574 include in situ external dose rate measurements using TLDs at BLANCA.

The population of interest to resolve the decisions includes the spatial boundaries of the sites. This spatial boundary includes, at a minimum, the area(s) of radioactive contamination that could cause a potential dose above action levels, and may include an area as large as the entire watershed that encompasses the site.

The data quality indicators (DQIs) of precision, accuracy, representativeness, completeness, comparability, and sensitivity needed to satisfy DQO requirements are discussed in Section 7.0. Data will be assessed in the CR to confirm or refute the CSM and determine whether the DQO data needs were met. Data collection and analysis methods must be capable of measuring analyte concentrations at or below the corresponding action levels. As appropriate, detection limits will be determined and compared to the action levels described in Section 3.2.1. If detection limits are higher than action levels, the detection limit will be used to make closure decisions. 
The DQO process specifies performance criteria for the decision rules. Setting tolerable limits on decision errors requires the planning team to weigh the relative effects of threats to human health and the environment, expenditure of resources, and the consequences of an incorrect decision. In general, confidence in DQO decisions will be established by the following:

- Developing a CSM

- Testing the validity of the CSM based on data analysis

- Evaluating the quality of the data based on DQI parameters

\subsection{RESULTS OF THE DQO ANALYSIS}

The problem statement, "Existing information is insufficient to determine the extent of contamination and confirm closure of CAU 574," will be resolved through an evaluation of available characterization data and the collection and evaluation of additional data to define the extent of contamination that exceeds the FAL.

At NEPTUNE, available characterization data are sufficient to resolve the decisions by calculating internal and external dose rates to determine the TED rates, comparing the TED rates to the FAL, defining the extent of contamination exceeding the FAL, and delineating a UR boundary.

At BLANCA, additional data will be collected with TLDs to determine the external dose rate component of TED at the site, and available characterization data will be used to calculate internal dose rates. The TED rates will then be determined and compared to the FAL, the extent of contamination exceeding the FAL will be defined, and a UR boundary will be delineated.

\subsubsection{Action Level Determination and Basis}

The action levels for radiological contaminants are based on the screening limits recommended in the National Council on Radiation Protection (NCRP) Report No. 129 for construction, commercial, industrial land use scenarios (NCRP, 1999) using a 25-mrem/yr dose constraint (Murphy, 2004) and the generic guidelines for residual concentration of radionuclides in DOE O 5400.5 (DOE, 1993).

The FAL for CAU 574 is a TED rate of 25 mrem/yr based on the Occasional Use Area exposure scenario, which is defined as workers being exposed to the site occasionally, up to 80 hours per year for 5 years. The TED rate is determined by summing the internal and external dose components.

The Residual Radioactive (RESRAD) computer code has been used to derive RRMGs for the internal dose component for each radionuclide. The RRMGs are the activity concentrations of individual radionuclides in surface soil that would cause a receptor to receive an internal dose equal to the FAL of $25 \mathrm{mrem} / \mathrm{yr}$ under the conditions described in a given exposure scenario. The CAU 551 soil sample results listed in Table 1 will be compared to the RRMGs using the sum of the fractions approach to calculate internal dose rates at NEPTUNE.

Table 2 lists the RRMGs calculated for each exposure scenario (Industrial Area, Remote Work Area, and Occasional Use Area). The RESRAD code variables (i.e., input parameters) used to calculate the RRMGs are listed in Table 3. 
TABle 2. RESIDUAL RADIOACTIVE MATERIAL GUIDElines DeRIVED FOR CORRECTIVE ACTION UNIT 574

\begin{tabular}{|c|c|c|c|c|c|c|}
\hline \multirow{2}{*}{ Radionuclide } & \multicolumn{5}{|c|}{ RRMG (pCi/g) } \\
\cline { 2 - 7 } & \multicolumn{3}{|c|}{ NEPTUNE } & \multicolumn{3}{c||}{ BLANCA } \\
\cline { 2 - 7 } & Industrial & $\begin{array}{c}\text { Remote } \\
\text { Work }\end{array}$ & $\begin{array}{c}\text { Occasional } \\
\text { Use }\end{array}$ & Industrial & $\begin{array}{c}\text { Remote } \\
\text { Work }\end{array}$ & $\begin{array}{c}\text { Occasional } \\
\text { Use }\end{array}$ \\
\hline \hline americium-241 & 350 & 1,334 & 5,611 & 341 & 1,281 & 5,387 \\
\hline cobalt-60 & 98,590 & 600,300 & $2,525,000$ & 105,800 & 652,500 & $2,744,000$ \\
\hline cesium-137 & 53,650 & 355,900 & $1,497,000$ & 58,490 & 401,100 & $1,687,000$ \\
\hline europium-152 & 323,200 & $1,615,000$ & $6,793,000$ & 332,700 & $1,638,000$ & $6,891,000$ \\
\hline europium-154 & 228,800 & $1,172,000$ & $4,928,000$ & 236,800 & $1,198,000$ & $5,037,000$ \\
\hline europium-155 & $1,502,000$ & $7,835,000$ & $32,950,000$ & $1,561,000$ & $8,055,000$ & $33,880,000$ \\
\hline plutonium-238 & 398 & 1,516 & 6,378 & 383 & 1,434 & 6,030 \\
\hline plutonium-239 & 360 & 1,376 & 5,788 & 347 & 1,302 & 5,475 \\
\hline plutonium-240 & 360 & 1,376 & 5,788 & 347 & 1,302 & 5,475 \\
\hline strontium-90 & 16,410 & 99,480 & 418,400 & 17,770 & 109,000 & 458,600 \\
\hline \hline
\end{tabular}

$\mathrm{pCi} / \mathrm{g}=$ picocurie(s) per gram

RRMG = Residual Radioactive Material Guideline 
TABle 3. Residual Radioactive Computer Code InPut Parameters

\begin{tabular}{|c|c|c|c|c|c|c|c|c|}
\hline \multirow{3}{*}{ Parameter } & \multirow{3}{*}{ Unit } & \multicolumn{6}{|c|}{ Parameter Value } & \multirow{3}{*}{ Comments/Basis } \\
\hline & & \multicolumn{2}{|c|}{ Industrial } & \multicolumn{2}{|c|}{ Remote Work } & \multicolumn{2}{|c|}{ Occasional Use } & \\
\hline & & NEPTUNE & BLANCA & NEPTUNE & BLANCA & NEPTUNE & BLANCA & \\
\hline Area of CZ & $\mathrm{m}^{2}$ & 70,000 & 680,000 & 70,000 & 680,000 & 70,000 & 680,000 & $\begin{array}{l}\text { Based on actual area. RESRAD is } \\
\text { sensitive to differences in area in the range } \\
\text { of the default value selected. The effect } \\
\text { on using the actual site area is to lower the } \\
\text { RRMGs. }\end{array}$ \\
\hline Thickness of CZ & $\mathrm{m}$ & 1.0 & 0.05 & 1.0 & 0.05 & 1.0 & 0.05 & $\begin{array}{l}\text { NEPTUNE based on CAU } 551 \text { data; } \\
\text { BLANCA based on Soils Sub-Project } \\
\text { assumptions. }\end{array}$ \\
\hline Density of CZ & $\mathrm{g} / \mathrm{cm}^{3}$ & 1.5 & 1.5 & 1.5 & 1.5 & 1.5 & 1.5 & NNSA/NSO, 2006b \\
\hline CZ Erosion Rate & $\mathrm{m} / \mathrm{yr}$ & 0.001 & 0.001 & 0.001 & 0.001 & 0.001 & 0.001 & NNSA/NSO, 2006b \\
\hline CZ Total Porosity & & 0.4 & 0.4 & 0.4 & 0.4 & 0.4 & 0.4 & NNSA/NSO, 2006b \\
\hline CZ Field Capacity & & 0.2 & 0.2 & 0.2 & 0.2 & 0.2 & 0.2 & NNSA/NSO, 2006b \\
\hline $\begin{array}{l}\text { CZ Hydraulic } \\
\text { Conductivity }\end{array}$ & $\mathrm{m} / \mathrm{yr}$ & 10 & 10 & 10 & 10 & 10 & 10 & NNSA/NSO, 2006b \\
\hline CZ b Parameter & & 5.3 & 5.3 & 5.3 & 5.3 & 5.3 & 5.3 & NNSA/NSO, 2006b \\
\hline $\begin{array}{l}\text { Average Annual } \\
\text { Wind Speed }\end{array}$ & $\mathrm{m} / \mathrm{s}$ & 4.07 & 4.07 & 4.07 & 4.07 & 4.07 & 4.07 & NNSA/NSO, 2006b \\
\hline $\begin{array}{l}\text { Evapotranspiration } \\
\text { Coefficient }\end{array}$ & & 0.5 & 0.5 & 0.5 & 0.5 & 0.5 & 0.5 & NNSA/NSO, 2006b \\
\hline Precipitation & $\mathrm{m} / \mathrm{yr}$ & 0.1626 & 0.1626 & 0.1626 & 0.1626 & 0.1626 & 0.1626 & Site-specific \\
\hline Runoff Coefficient & & 0.4 & 0.4 & 0.4 & 0.4 & 0.4 & 0.4 & NNSA/NSO, 2006b \\
\hline Inhalation Rate & $\mathrm{m}^{3} / \mathrm{yr}$ & 8,400 & 8,400 & 12,300 & 12,300 & 12,300 & 12,300 & NNSA/NSO, 2006b \\
\hline $\begin{array}{l}\text { Mass Loading for } \\
\text { Inhalation }\end{array}$ & $\mathrm{g} / \mathrm{m}^{3}$ & 0.0006 & 0.0006 & 0.0006 & 0.0006 & 0.0006 & 0.0006 & NNSA/NSO, 2006b \\
\hline Exposure Duration & $\mathrm{yr}$ & 25 & 25 & 25 & 25 & 25 & 25 & NNSA/NSO, 2006b \\
\hline $\begin{array}{l}\text { Shielding Factor } \\
\text { Inhalation }\end{array}$ & & 0.4 & 0.4 & 0.4 & 0.4 & 0.4 & 0.4 & $\begin{array}{l}\text { RESRAD default is based on typical } \\
\text { building construction and ventilation } \\
\text { methods. }\end{array}$ \\
\hline $\begin{array}{l}\text { Shielding Factor } \\
\text { External Gamma }\end{array}$ & & N/A & $\mathrm{N} / \mathrm{A}$ & $\mathrm{N} / \mathrm{A}$ & N/A & $\mathrm{N} / \mathrm{A}$ & $\mathrm{N} / \mathrm{A}$ & $\begin{array}{l}\text { Not applicable; external gamma pathway } \\
\text { is not in use. }\end{array}$ \\
\hline
\end{tabular}


Table 3. Residual Radioactive Computer Code InPut Parameters (CONTINued)

\begin{tabular}{|c|c|c|c|c|c|c|c|c|}
\hline \multirow{3}{*}{ Parameter } & \multirow{3}{*}{ Unit } & \multicolumn{6}{|c|}{ Parameter Value } & \multirow{3}{*}{ Comments/Basis } \\
\hline & & \multicolumn{2}{|c|}{ Industrial } & \multicolumn{2}{|c|}{ Remote Work } & \multicolumn{2}{|c|}{ Occasional Use } & \\
\hline & & NEPTUNE & BLANCA & NEPTUNE & BLANCA & NEPTUNE & BLANCA & \\
\hline $\begin{array}{l}\text { Fraction of Time } \\
\text { Spent Indoors }\end{array}$ & & 0.171 & 0.171 & N/A & N/A & N/A & N/A & $\begin{array}{l}\text { Industrial is based on current Soils } \\
\text { Sub-Project procedures and assumptions; } \\
\text { Remote Work and Occasional Use assume } \\
\text { all hours are outdoors. }\end{array}$ \\
\hline $\begin{array}{l}\text { Fraction of Time } \\
\text { Spent Outdoors }\end{array}$ & & 0.0856 & 0.0856 & 0.0384 & 0.0384 & 0.00913 & 0.00913 & $\begin{array}{l}\text { Industrial is based on current Soils } \\
\text { Sub-Project procedures and assumptions; } \\
\text { Remote Work and Occasional Use assume } \\
\text { all hours are outdoors. }\end{array}$ \\
\hline Shape Factor & & 1 & 1 & 1 & 1 & 1 & 1 & Assumed circular deposition. \\
\hline Soil Ingestion Rate & $\mathrm{g} / \mathrm{yr}$ & 36.5 & 36.5 & 36.5 & 36.5 & 36.5 & 36.5 & $\begin{array}{l}\text { RESRAD applies occupancy factor } \\
\text { corrections to this value through } \\
\text { calculation; the value should not be } \\
\text { corrected prior to input (NNSA/NSO, } \\
\text { 2006b [ } 480 \mathrm{mg} / \text { day]). }\end{array}$ \\
\hline Depth of Mixing & $\mathrm{m}$ & .15 & .056 & .15 & .056 & .15 & .056 & $\begin{array}{l}\text { RESRAD default for NEPTUNE due to } \\
\text { man-made disturbance of the area; } \\
\text { BLANCA relies upon Soils Sub-Project } \\
\text { assumption that } 90 \text { percent of } \\
\text { contaminants are in the top } 5 \mathrm{~cm} \text { of soil. }\end{array}$ \\
\hline
\end{tabular}

CAU $=$ Corrective Action Unit $\mathrm{cm}=$ centimeter(s)

$\mathrm{CZ}=$ contamination zone

$\mathrm{g} / \mathrm{cm}^{3}=$ gram(s) per cubic centimeter

$\mathrm{g} / \mathrm{m}^{3}=$ gram(s) per cubic meter

$\mathrm{g} / \mathrm{yr}=\operatorname{gram}(\mathrm{s})$ per year

$\mathrm{m}=$ meter(s)

$\mathrm{m}^{2}=$ square meter(s)

$\mathrm{m}^{3} / \mathrm{yr}=$ cubic meter(s) per year $\mathrm{m} / \mathrm{s}=$ meter(s) per second

$\mathrm{m} / \mathrm{yr}=$ meter(s) per year

$\mathrm{mg} /$ day = milligram(s) per day

NNSA/NSO = U.S. Department of Energy, National Nuclear Security Administration Nevada Site Office

N/A = not applicable

RESRAD = Residual Radioactive

RRMG = Residual Radioactive Material Guideline

$\mathrm{yr}=$ year $(\mathrm{s})$ 


\subsubsection{Hypothesis Test}

The baseline condition (i.e., null hypothesis) and alternative condition are:

- Baseline condition - closure objectives have not been met.

- Alternative condition - closure objectives have been met.

The closure objective is to define the extent of contamination that exceeds the FAL to define a UR. Sufficient evidence to reject the null hypothesis is the identification of the extent of contamination.

\subsubsection{Statistical Model}

A judgmental measurement scheme will be implemented to select in situ external dose rate measurement (TLD) locations at BLANCA and evaluate DQO decisions. Each measurement will be compared to fixed threshold values (i.e., FALs) on a point-by-point basis to determine the appropriate resolution to the decisions; therefore, a statistical model does not apply to the measurement scheme for CAU 574.

\subsubsection{Design Description/Option}

A judgmental design will be used to select locations for collection of in situ external dose rate measurements at BLANCA. Because individual measurements, rather than an average, will be compared to the FAL, statistical methods will not be used. Adequate representativeness of the entire target population is not a requirement because good prior information is available for BLANCA, as discussed in Section 2.2.4. Therefore, the measurement scheme is designed to collect measurements from areas known to have the highest levels of contamination. If the observed measurements are below the action level, then a decision can be made without the samples being truly representative of the entire area (EPA, 2006).

In situ external dose rate measurement (TLD) locations will be selected to satisfy the DQI of representativeness in that selected locations will best represent the populations of interest and target areas with the highest potential for contamination. TLD locations will be determined based on process knowledge and the results of the 1994 aerial radiological (flyover) survey. Section 4.2.2.1 provides additional details on the collection of external dose rate measurements and the placement of TLDs at BLANCA.

\subsubsection{Conceptual Site Model}

The CSM describes the most probable scenario for current conditions at the site and defines the assumptions that are the basis for identifying the future land use, contaminant sources, release mechanisms, migration pathways, and exposure routes and for choosing a conceptual CAA. The CSM is based on physical setting, potential contaminant sources, release information, historical documentation, personnel interviews, process knowledge, site visits, photographs, field screening, and analytical results. The CSM was used to develop appropriate sampling strategies and data collection methods. The CSM for these sites is summarized in Table 4.

If evidence of contamination that is not consistent with the CSM is identified during CAI activities, the situation will be reviewed, the CSM will be revised, the DQOs will be reassessed, and a recommendation will be made as to how best to proceed. In such cases, participants in the DQO process will be notified and given the opportunity to comment on and/or concur with the recommendation. 
Table 4. Conceptual Site Model for Corrective Action Unit 574

\begin{tabular}{|c|c|c|}
\hline CSM Element & $\begin{array}{c}\text { CAS 12-23-10 } \\
\text { U12c.03 Crater (Neptune) }\end{array}$ & $\begin{array}{c}\text { CAS 12-45-01 } \\
\text { U12e.05 Crater (Blanca) }\end{array}$ \\
\hline Site Status & \multicolumn{2}{|l|}{ Inactive and/or abandoned } \\
\hline Land Use Zone & \multicolumn{2}{|c|}{$\begin{array}{l}\text { Nuclear Test Zone: This area is reserved for dynamic experiments, } \\
\text { hydrodynamic tests, and underground nuclear weapons and weapons effects } \\
\text { tests. This zone includes compatible defense and nondefense research, } \\
\text { development, and testing activities. }\end{array}$} \\
\hline Exposure Scenario & \multicolumn{2}{|c|}{$\begin{array}{l}\text { Occasional Use Area: Worker will be exposed to the site occasionally (up to } \\
80 \text { hours per year for } 5 \text { years). Site structures are not present for shelter and } \\
\text { comfort of the worker. }\end{array}$} \\
\hline $\begin{array}{l}\text { Sources of Potential Soil } \\
\text { Contamination }\end{array}$ & \multicolumn{2}{|c|}{ Fallout and soil activation from venting of underground nuclear testing } \\
\hline Impacted Media & \multicolumn{2}{|l|}{ Surface and subsurface soil } \\
\hline Potential Contaminants & \multicolumn{2}{|l|}{ Radionuclides } \\
\hline Extent of Contamination & \multicolumn{2}{|c|}{$\begin{array}{l}\text { Contamination, if present, is expected to be contiguous to the release points. } \\
\text { Concentrations are expected to decrease with distance from the source. } \\
\text { Groundwater contamination is not expected. }\end{array}$} \\
\hline Transport Mechanisms & \multicolumn{2}{|c|}{$\begin{array}{l}\text { Surface water runoff may provide for the transportation of some } \\
\text { contaminants within or outside of the boundaries of the CASs. There is a } \\
\text { known watershed at the CASs. Infiltration of precipitation through } \\
\text { subsurface media serves as a minor driving force for migration of } \\
\text { contaminants. Ejection of material from the craters provided a transport } \\
\text { mechanism at detonation. }\end{array}$} \\
\hline Migration Pathways & \multicolumn{2}{|c|}{$\begin{array}{l}\text { Lateral transport (runoff) is expected to dominate over vertical transport } \\
\text { (infiltration) due to limited vertical infiltration. The sites are located within } \\
\text { a known watershed that collects down slope to the southeast either within } \\
\text { the existing UR for CAU } 551 \text { or in the Radioactive Material Area located to } \\
\text { the southeast of the existing UR. The area is subject to infrequent, } \\
\text { potentially intense, stormwater flows that provide an intermittent mechanism } \\
\text { for both vertical and horizontal transport of contaminants. Contaminated } \\
\text { sediments entrained by these stormwater events would be carried by the } \\
\text { streamflow to locations where the flowing water loses energy and the } \\
\text { sediments drop out. }\end{array}$} \\
\hline $\begin{array}{l}\text { Lateral and Vertical Extent of } \\
\text { Contamination }\end{array}$ & \multicolumn{2}{|c|}{$\begin{array}{l}\text { Subsurface contamination is assumed to be present in the NEPTUNE crater } \\
\text { and in the location where the BLANCA test vented. Contamination is } \\
\text { expected to be contiguous to the release points. Concentrations are expected } \\
\text { to decrease with distance and depth from the source. Groundwater } \\
\text { contamination is not expected. Lateral and vertical extent of COC } \\
\text { contamination is assumed to be within the spatial boundaries of the CAS. }\end{array}$} \\
\hline Exposure Pathways & \multicolumn{2}{|c|}{$\begin{array}{l}\text { The potential for contamination exposure is limited to industrial and } \\
\text { construction workers, and military personnel conducting training. These } \\
\text { human receptors may be exposed through inhalation or incidental ingestion } \\
\text { of soil due to inadvertent disturbance of materials or through irradiation by } \\
\text { radioactive materials. }\end{array}$} \\
\hline
\end{tabular}


CAU 574 SAFER Plan

Section: Data Quality Objectives

Revision: 0

Date: August 2011

THIS PAGE INTENTIONALLY LEFT BLANK 


\subsection{FIELD ACTIVITIES AND CLOSURE OBJECTIVES}

This section provides a description of the field activities and closure objectives for CAU 574.

\subsection{Contaminants of Potential Concern}

Contaminants of potential concern (COPCs) were identified through a review of site history, process knowledge, personal interviews, and past investigation efforts. The list of COPCs is intended to encompass all of the significant contaminants that could potentially be present at each CAS. Significant contaminants are defined as COPCs that exceed action levels.

\subsubsection{Corrective Action Site 12-23-10, U12c.03 Crater (Neptune)}

The targeted contaminants at NEPTUNE are plutonium and americium, which contribute to internal dose. This is based on both operational history and historical data from this and other safety experiments. Some fission products are also present that may contribute to external dose; however, the primary decision driver at this site is expected to be the internal dose component.

\subsubsection{Corrective Action Site 12-45-01, U12e.05 Crater (Blanca)}

The targeted contaminants at BLANCA are fission products. This is based on both operational history and historical data from this and other weapons-related experiments in which a fission event occurred. Fission products include the higher-level gamma emissions that lead to external dose. The external dose component is expected to be the decision driver at this site.

Americium-241 was not detected during the 1994 flyover survey; therefore, it can be assumed that the internal dose component does not significantly contribute to the TED at this site.

\subsection{REMEDIATION}

The DQOs developed for CAU 574 identified data gaps that require additional data collection before identifying and implementing the preferred closure alternative. A decision point approach, based on the DQOs, for making remediation decisions is summarized in Figure 3. Decision points that require a consensus between NNSA/NSO and NDEP before continuing are identified in Figure 3. The closure strategy for CAU 574 under this SAFER process is discussed in the following sections.

\subsubsection{Corrective Action Site 12-23-10, U12c.03 Crater (Neptune)}

A large body of historical information is available for NEPTUNE, as discussed in Section 2.1.4. The available existing characterization data will be used to resolve the decisions and delineate the extent of contamination that exceeds the FAL to define a UR at this site. Additional data will not be collected for NEPTUNE.

\subsubsection{Calculate External Dose Rates}

External dose rates at NEPTUNE will be determined by RIDP data correlated to 1994 flyover survey data, as summarized in Section 2.1.4.3. This method is described in detail and validated in Radionuclide Inventory Distribution Project Data Evaluation and Verification White Paper (NSTec, 2008). External dose rate calculations will be very conservative, and because external dose is not the primary decision driver at this site, this approach is justified. 


\subsubsection{Calculate Internal Dose Rates}

Date: August 2011

Internal dose rates at NEPTUNE will be determined using the CAU 551 soil sample results listed in Table 1 and RIDP data correlated to the 1994 flyover survey data. In the locations where soil samples were collected for CAU 551, the soil sample data will be compared to the RRMGs listed in Table 2 using the sum of the fractions approach to calculate internal dose rates. In locations outside the existing UR for CAU 551 where soil samples were not collected and where americium-241 was detected during the 1994 flyover survey, RIDP data correlated to the 1994 flyover survey data will be used to calculate internal dose rates.

As identified in the CSM for this site, a migration pathway exists due to runoff that collects down slope to the southeast of the site within the existing UR for CAU 551. The area is subject to infrequent, potentially intense, stormwater flows that provide an intermittent mechanism for transport of contaminants. This assumption is verified by the soil sample results for CAU 551. Soil samples collected along the wash to the southeast of the americium plume show that there are elevated concentrations of americium and plutonium along this drainage path that were not detected during the 1994 flyover survey. Therefore, the soil sample data collected for CAU 551 in this area provide an excellent source for calculation of internal dose in an area where a correlation to 1994 flyover survey data would not be useful. Results of soil samples collected along the road to the southeast of the existing UR for CAU 551 will also be analyzed to verify that contamination above the FAL is not present outside the existing UR for CAU 551. The CAU 551 soil sample data are sufficient to characterize areas of potential migration outside of the americium-241 plume that was identified by the 1994 flyover survey.

To the northwest and southwest of the existing UR for CAU 551 where americium-241 was detected during the 1994 flyover survey, soil samples were not collected for characterization of CAU 551. In these areas, RIDP data correlated to the 1994 flyover survey data will be used to calculate internal dose rates. As summarized in Section 2.1.4.3, these calculations will take a very conservative approach to calculate internal dose rates (NSTec, 2008).

If the two methods of determining internal dose rates (i.e., CAU 551 soil sample results and RIDP data correlated to the 1994 flyover survey data) produce significantly different results, the more conservative of the two approaches will be applied across the site.

\subsubsection{Define Use Restriction Boundaries}

The TED will be determined by summing the internal and external dose components. Decision criteria will be based on the 95-percent upper confidence limit of the average TED estimates. The 25-mrem/yr boundary will be delineated for each exposure scenario (Industrial Area, Remote Work Area, and Occasional Use Area), and appropriate URs will be established.

Calculation of TED and establishment of UR boundaries will be conservative. Due to the remote location of the site and limited use and accessibility of the area, a less precise, more conservative UR boundary is justified.

\subsubsection{Corrective Action Site 12-45-01, U12e.05 Crater (Blanca)}

For BLANCA, additional data will be collected to resolve the decisions and delineate the extent of contamination that exceeds the FAL to define a UR. Available existing characterization data will also be used to validate assumptions and provide a layer of conservatism to the decisions. Additional details and justification for this approach are provided in the following sections. 


\subsubsection{Collect In Situ External Dose Rate Measurements}

At BLANCA, external dose rates will be determined by collecting in situ measurements using TLDs. TLD locations will be based judgmentally on the results of the 1994 flyover survey data and correlated NNSS-wide RIDP data. A transect that is approximately normal to the flyover survey isopleths will be established at BLANCA, and TLDs will be placed along this transect.

At least one TLD will be placed at a location where the TED is expected to be less than the FAL. To meet this constraint, it will be necessary to determine preliminary 25 -mrem/yr boundaries. These preliminary calculations will be accomplished by plotting the dose rate estimates from the correlation of RIDP data to the 1994 flyover survey data. This evaluation will be augmented with historical environmental monitoring TLD dose rate measurements and recent Soils Sub-Project site closure data.

If it is determined based on actual TLD measurements that at least one TLD had not been placed outside the 25-mrem/yr boundary, the extent of contamination that exceeds the FAL will not have been identified, and additional TLDs will be placed to define the extent of contamination exceeding the FAL.

Background radiation will be subtracted from the TLD measurements using results of the 1994 flyover survey. As discussed in Section 2.1.4.1, measurements of background radiation were continuously collected during the 1994 flyover survey. These background measurements will be subtracted at each TLD location.

\subsubsection{Calculate Internal Dose Rates}

Americium-241 was not detected at BLANCA during the 1994 flyover survey; therefore, it is assumed that the internal dose component does not significantly contribute to TED at this site. Soil samples to quantify this small component of TED will not be collected. Instead, a conservative approach will be taken using RIDP data. Because internal dose is not the primary decision driver at this site, this approach is justified.

Although RIDP data were not collected in the vicinity of BLANCA, RIDP data, when averaged across associated 1994 flyover isopleths, are well correlated to the 1994 flyover survey data (NSTec, 2008). RIDP data from areas with the same level of contamination as that detected at BLANCA in the 1994 flyover survey will be used to calculate internal dose rates. The highest RIDP value for non-americium gamma emitters and inferred strontium-90 will be used to calculate the internal dose rate for application at all TLD locations at BLANCA.

\subsubsection{Define Use Restriction Boundaries}

The TED will be determined by summing the internal and external dose components. Decision criteria will be based on the 95-percent upper confidence limit of the average TED estimates. The 25-mrem/yr boundary will be delineated for each exposure scenario (Industrial Area, Remote Work Area, and Occasional Use Area), and appropriate URs will be established.

Calculation of TED and establishment of UR boundaries will be conservative. Due to the remote location of the site and limited use and accessibility of the area, a less precise, more conservative UR boundary is justified. 


\subsection{VERIFICATION}

The closure objectives for CAU 574 will have been met when the extent of contamination exceeding the the FAL has been defined.

\subsection{Closure}

If contamination is not present at levels above the FAL at a site, the site will be closed with no further action. If contamination is present at levels above the FAL, the site will be closed in place with a UR that will provide adequate administrative controls to limit site access and worker dose. The decision logic is provided in Figure 3.

\subsection{DURATION}

Table 5 provides a tentative duration of SAFER activities in calendar days.

Table 5. Project Schedule for Corrective Action Unit 574

\begin{tabular}{|l|c|}
\hline \multicolumn{1}{|c|}{ Activity } & Duration (calendar days) \\
\hline \hline Existing data analysis & 20 \\
\hline TLD installation & 10 \\
\hline TLD data acquisition, validation, and assessment & 120 \\
\hline Closure Report development & 200 \\
\hline
\end{tabular}




\subsection{REPORTS AND RECORDS AVAILABILITY}

Reports generated during field activities will be provided to NDEP upon request. Historical information and documents referenced in this document are retained in the NNSA/NSO project files in Las Vegas, Nevada, and can be obtained through written request to the NNSA/NSO Federal Sub-Project Director. This document is available in the DOE public reading rooms located in Las Vegas and Carson City, Nevada, or by contacting the appropriate NNSA/NSO Federal Sub-Project Director. 
CAU 574 SAFER Plan

Section: Reports and Records

Revision: 0

Date: August 2011

\section{THIS PAGE INTENTIONALLY LEFT BLANK}




\subsection{INVESTIGATION/REMEDIATION WASTE MANAGEMENT}

This project is not expected to generate any investigation or remediation waste. 
CAU 574 SAFER Plan

Section: Waste Management

Revision: 0

Date: August 2011

\section{THIS PAGE INTENTIONALLY LEFT BLANK}




\subsection{QUALITY ASSURANCE/QUALITY CONTROL}

The overall objective of the characterization activities described in this SAFER Plan is to collect accurate and defensible data to support the selection and implementation of a closure alternative for CAU 574.

\subsection{SAMPle Collection Activities}

The data from the TLD measurements will meet rigorous data quality requirements. The TLDs will be obtained from, and measured by, the NSTec Environmental Technical Services group. This group is responsible for the routine environmental monitoring program at the NNSS, and the same approach will be used at CAU 574 (NNSA/NSO, 2010).

Quality assurance procedures for TLD monitoring of ambient radiation involve comparing the data from paired TLDs at each location to estimate measurement precision, comparing current and past measurements at each location, and reviewing data from the TLDs in control locations. The average relative percent difference between pairs of environmental TLDs was 2.3 percent for 2009 (NNSA/NSO, 2010).

The TLDs will be submitted to the Environmental Technical Services group and analyzed using automated TLD readers that are calibrated and maintained by the NSTec Radiological Control Department in accordance with existing quality control procedures for TLD processing. Certification is maintained through the DOE Laboratory Accreditation Program for dosimetry.

The determination of the external dose component of the TED by TLDs was determined to be the most accurate method because:

- The TLDs will be exposed for the 2,250 hours of exposure time used for the Industrial Area exposure scenario. This eliminates errors in reading dose rate meter scale graduations and needle fluctuations. These errors would be magnified when as-read meter values are multiplied from units of "per-hour" to 2,250 hours.

- The use of a TLD to determine an individual's external exposure is the standard in radiation safety and serves as the legal dose of record when other measurements are available. Specifically, 10 CFR 835 indicates that personal dosimeters shall be provided to monitor individual exposures and that the monitoring program that uses the dosimeters shall be accredited in accordance with a DOE Laboratory Accreditation Program (CFR, 1993).

\subsection{APPLICABLE LABORATORY/ANALYTICAL DATA QUALITY INDICATORS}

The Corrective Action Decision Document/Closure Report for CAU 551 discusses the results of the data quality assessment for soil sample data (NNSA/NSO, 2006a). To ensure that the dataset was of sufficient quality and completeness, the entire dataset, as well as individual sample results, were assessed against the DQIs of precision, accuracy, comparability, completeness, and representativeness, as defined in the Industrial Sites Quality Assurance Project Plan (U.S. Department of Energy, National Nuclear Security Administration Nevada Operations Office, 2002). No rejected data for radionuclides were generated during the CAI for CAU 551. 
A data quality assessment of the RIDP data has also been completed (NSTec, 2008). Precision, accuracy, representativeness, completeness, and comparability were used to evaluate data quality. The data quality was determined to be adequate for determining conservative 25-mrem/yr dose rate boundaries and planning remedial actions.

The following sections discuss the DQIs that have been evaluated for existing data.

\subsubsection{Precision}

Precision is used to assess the variability of a population of measurements with the variability of the analysis process through the use of matrix spike/matrix spike duplicates, laboratory duplicates, laboratory control sample/laboratory control sample duplicates, and field duplicate samples. Precision for radiological analysis is conducted by evaluating the duplicate precision through the relative percent difference (RPD) or normalized difference (ND). The RPD is applicable when both the sample and its duplicate have concentrations of the target radionuclide exceeding five times the minimum detectable concentration (MDC). This excludes many measurements because the samples contain non-detectable levels of the target radionuclide. In situations where the RPD does not apply, duplicate results are evaluated using the ND.

To determine data precision for CAU 551, all samples, including field quality control samples, were evaluated and incorporated into the precision calculation for CAU 551. Isotopic plutonium had an FD precision rate of 73.7 percent and an ND of 66.7 percent. With the source of plutonium being from a safety experiment, the plutonium would have been dispersed in particle form, thus the appearance of a particle in duplicates is a reasonable result. Therefore, the dataset for CAU 551 was determined to be acceptable for the DQI of precision.

\subsubsection{Accuracy}

Accuracy is a measure of the closeness of an individual measurement or the average or a number of measurements to the true value. It is used to assess the performance of laboratory measurement processes as well as to evaluate individual groups of analyses.

To determine accuracy for CAU 551, all samples, including field quality control samples, were evaluated and incorporated into the accuracy calculation for CAU 551. The radiological accuracy measurements exceed 85 percent, thereby meeting the performance metric of an accuracy of at least 80 percent. The dataset for CAU 551 was determined to be acceptable for the DQI of accuracy.

\subsubsection{Representativeness}

Representativeness is the degree to which sample data accurately and precisely represent a characteristic of a population, parameter variations at a sampling point, or an environmental condition. Representativeness is assured by a carefully developed sampling strategy, collecting the specified number of samples from proper sampling locations, and analyzing them by the approved analytical methods.

During the DQO process for CAU 551, it was identified that representative samples could not be collected from all areas of the muckpiles due to safety considerations. However, the use of historical muckpile data was evaluated and determined to be sufficient to fill this potential data gap. The newly acquired data were collected from areas most likely to contain contamination. Therefore, the analytical data acquired for CAU 551 combined with the historical muckpile data were considered representative of the population parameters. 


\subsubsection{Comparability}

Comparability is a qualitative parameter expressing the confidence with which one dataset can be compared to another.

Field sampling for CAU 551 was performed and documented in accordance with approved procedures that are comparable to standard industry practices. DOE-approved analytical methods and procedures were used to analyze, report, and validate the data. These are comparable to other methods used not only in industry and government practices but, most importantly, are comparable to other investigations conducted for the NNSS. Therefore, datasets for CAU 551 were considered comparable to other datasets generated using these same standardized DOE procedures, thereby meeting DQO requirements.

Also, standard, approved field and analytical methods ensured that data were appropriate for comparison to the action levels specified for CAU 551.

\subsubsection{Completeness}

Completeness is a quantitative and qualitative evaluation of measurement system performance. The criterion for meeting completeness is defined as generating sufficient data of the appropriate quality to satisfy the data needs.

Acceptable criteria for completeness for CAU 551 were defined to be 80 percent of non-critical analytes as having valid results and 90 percent of critical analytes having valid results. Also, the data must be of sufficient quality to be able to support the DQO decisions. Rejected data would not be used in the resolution of DQO decisions for CAU 551 and would not be counted towards meeting the completeness goals. No rejected data were generated for CAU 551.

\subsubsection{Sensitivity}

The sensitivity goal states that analytical detection limits will be less than the corresponding action level, thus ensuring that the analyses were sensitive enough to identify and quantify contaminants present in the samples. Sample results for CAU 551 were assessed against the DQI of sensitivity. All radiological analytes met the sensitivity goal. 
Revision: 0

Date: August 2011

\section{THIS PAGE INTENTIONALLY LEFT BLANK}




\subsection{REFERENCES}

CFR, see Code of Federal Regulations.

Code of Federal Regulations, 1993. Title 10 CFR Part 835, “Occupational Radiation Protection.” Washington D.C.

Desert Research Institute, 1987. Hydrologeologic Investigations of Flow in Fractured Tuffs, Rainier Mesa, Nevada Test Site. Las Vegas, NV.

Desert Research Institute, 1988. CERCLA Preliminary Assessments of DOE's Nevada Operations Office, Nuclear Weapons Testing Areas. Las Vegas, NV.

Desert Research Institute, 1996. ER-12-1 Completion Report, Publication \#45120. Prepared for the U.S. Department of Energy, Nevada Operations Office. Las Vegas, NV.

DOE, see U.S. Department of Energy.

DOE/NV, see U.S. Department of Energy, Nevada Operations Office.

DRI, see Desert Research Institute.

EPA, see U.S. Environmental Protection Agency.

FFACO, see Federal Facility Agreement and Consent Order.

Federal Facility Agreement and Consent Order, 1996 (as amended March 2010). Agreed to by the State of Nevada; U.S. Department of Energy, Environmental Management; U.S. Department of Defense; and U.S. Department of Energy, Legacy Management.

Geological Society of America, 1968. Nevada Test Site. Boulder, CO.

Gilbert, R. O., E. H. Essington, D. N. Brady, P. G. Doctor, and L. L Eberhardt, 1977. "Statistical Activities during 1976 and the Design and Initial Analysis of Nuclear Site Studies.” In Transuranics in Desert Ecosystems. NVO-181. Las Vegas, NV.

Hendricks, T. J., and S. R. Riedhauser, 1999. An Aerial Radiological Survey of the Nevada Test Site. DOE/NV/11718--324. Prepared by Bechtel Nevada for the U.S. Department of Energy. Las Vegas, NV.

Johnson, G. W., and C. E. Violet, 1958. Phenomenology of Contained Nuclear Explosions. UCRL-5124 Rev. 1. University of California, Lawrence Radiation Laboratory. Livermore, CA.

McArthur, R. D., and J. F. Kordas, 1983. Nevada Test Site Radionuclide Inventory and Distribution Program: The Galileo Area. DOE/NV/10162--14. Prepared by Desert Research Institute. Las Vegas, NV.

McArthur, R. D., and J. F. Kordas, 1985. Nevada Test Site Radionuclide Inventory and Distribution Program: Report \#2, Areas 2 and 4. DOE/NV/10162--20. Prepared by Desert Research Institute. Las Vegas, NV.

McArthur, R. D., and S. W. Mead, 1987. Nevada Test Site Radionuclide Inventory and Distribution Program: Report \#3, Areas 3, 7, 8, 9, and 10. DOE/NV/10384--15. Prepared by Desert Research Institute. Las Vegas, NV. 


\subsection{REFERENCES (continued)}

McArthur, R. D., and S. W. Mead, 1988. Nevada Test Site Radionuclide Inventory and Distribution Program: Report \#4, Areas 18 and 20. DOE/NV/10384--22. Prepared by Desert Research Institute. Las Vegas, NV.

McArthur, R. D., and S. W. Mead, 1989. Nevada Test Site Radionuclide Inventory and Distribution Program: Report \#5, Areas 5, 11, 12, 15, 17, 18, 19, 25, 26, and 30. DOE/NV/10384--26. Prepared by Desert Research Institute. Las Vegas, NV.

McArthur, R. D., 1991. Radionuclides in Surface Soil at the Nevada Test Site. DOE/NV/10845--02. Prepared by Desert Research Institute. Las Vegas, NV.

Murphy, T., 2004. Nevada Division of Environmental Protection Letter to R. M. Bangerter, Jr. (NNSA/NSO). Review of Industrial Sites Project Document Guidance for Calculating Industrial Sites Project Remediation Goals for Radionuclides in Soil Using the Residual Radiation (RESRAD) Computer Code. November 19, 2004. Las Vegas, NV.

National Council on Radiation Protection, 1999. Recommended Screening Limits for Contaminated Surface Soil and Review of Factors Relevant to Site-Specific Studies. NCRP Report No. 129. Washington, D.C.

National Security Technologies, LLC, 2008. Radionuclide Inventory Distribution Project Data Evaluation and Verification White Paper. DOE/NV/25946--994. Las Vegas, NV.

National Security Technologies, LLC, 2010. Nevada Test Site Environmental Report 2009. DOE/NV/25946--1067. Prepared for the U.S. Department of Energy, National Nuclear Security Administration Nevada Site Office. Las Vegas, NV.

NCRP, see National Council on Radiation Protection and Measurements.

NNSA/NSO, see U.S. Department of Energy, National Nuclear Security Administration Nevada Site Office.

NSTec, see National Security Technologies, LLC.

Proctor, A. E., 1997. Aerial Radiological Surveys. DOE/NV/11718--127. Bechtel Nevada. Las Vegas, NV.

Ristvet, B., DTRA Archivist. Personal interview. April 12, 2011. Las Vegas, NV.

Shelton, A. V., M. D. Nordyke, and R. H. Goechermann, 1960. The Neptune Event; A Nuclear Explosive Cratering Experiment. UCRL-5766. University of California, Lawrence Radiation Laboratory. Livermore, CA.

Tamura, T., 1977. "Plutonium Distribution in a Desert Pavement-Desert Mound Soil System in Area 11.” In Environmental Plutonium on the Nevada Test Site and Environs. NVO-171. Las Vegas, NV.

USGS, see U.S. Geological Survey.

U.S. Department of Energy, 1993. Radiation Protection of the Public and the Environment, DOE Order 5400.5, Change 2. Washington, D.C.

U.S. Department of Energy, National Nuclear Security Administration Nevada Operations Office, 2002. Industrial Sites Quality Assurance Project Plan, Nevada Test Site, Nevada. DOE/NV--372--REV. 3. Las Vegas, NV 


\subsection{REFERENCES (continued)}

U.S. Department of Energy, National Nuclear Security Administration Nevada Site Office, 2004. Corrective Action Investigation Plan for Corrective Action Unit 551: Area 12 Muckpiles, Nevada Test Site, Nevada. DOE/NV--976. Las Vegas, NV.

U.S. Department of Energy, National Nuclear Security Administration Nevada Site Office, 2006a. Corrective Action Decision Document/Closure Report for Corrective Action Unit 551: Area 12 Muckpiles, Nevada Test Site, Nevada. DOE/NV--1174-REV 1. Las Vegas, NV.

U.S. Department of Energy, National Nuclear Security Administration Nevada Site Office, 2006b. Industrial Sites Project Establishment of Final Action Levels. DOE/NV--1107, Rev. 0. Las Vegas, NV.

U.S. Department of Energy, Nevada Operations Office, 2000. United States Nuclear Tests, July 1945 through September 1992. DOE/NV--209-Rev 15. Las Vegas, NV.

U.S. Environmental Protection Agency, 2006. Guidance on Systematic Planning Using the Data Quality Objectives Process. EPA QA/G-4. Washington, D.C.

U.S. Geological Survey, 1965. Perched Ground Water in Zeolitized-Bedded Tuff, Rainier Mesa and Vicinity, Nevada Test Site, Nevada. USGS Report TEI-862. Las Vegas, NV.

U.S. Geological Survey, 1996. Summary of Hydrogeological Controls on Groundwater Flow at the Nevada Test Site, Nye County, Nevada. USGS WRIR 96-4109. Las Vegas, NV.

U.S. Geological Survey and U.S. Department of Energy, 2003. “USGS/DOE Nevada Well ER12-1 Site Page.” Las Vegas, NV.

Winograd, I. J., and W. Thordarson, 1975. Hydrology and Hydrochemical Framework, SouthCentral Great Basin, Nevada-California, with Special Reference to the Nevada Test Site. USGS Professional Paper 712-C. Denver, CO. 
Revision: 0

Date: August 2011

THIS PAGE INTENTIONALLY LEFT BLANK 
Revision: 0

Date: August 2011

\section{APPENDIX A. PROJECT ORGANIZATION}


CAU 574 SAFER Plan

Section: Appendix A

Revision: 0

Date: August 2011

\section{THIS PAGE INTENTIONALLY LEFT BLANK}




\section{PROJECT ORGANIZATION}

The U.S. Department of Energy, National Nuclear Security Administration Nevada Site Office Federal Soils Sub-Project Director is Kevin J. Cabble, and his telephone number is (702) 295-5000.

The identification of the project Health and Safety Officer and the Quality Assurance Officer can be found in the appropriate plan. However, personnel are subject to change, and it is suggested that the appropriate U.S. Department of Energy Federal Sub-Project Director be contacted for further information. The Task Manager will be identified in the Federal Facility Agreement and Consent Order Monthly Activity Report prior to the start of field activities. 
CAU 574 SAFER Plan

Section: Appendix A

Revision: 0

Date: August 2011

THIS PAGE INTENTIONALLY LEFT BLANK 
Revision: 0

Date: August 2011

\section{APPENDIX B. EVALUATION OF CORRECTIVE ACTION ALTERNATIVES}


CAU 574 SAFER Plan

Section: Appendix B

Revision: 0

Date: August 2011

THIS PAGE INTENTIONALLY LEFT BLANK 


\section{B.1.0 EVALUATION OF CORRECTIVE ACTION ALTERNATIVES}

This appendix presents the corrective action objectives for CAU 574, describes the general standards and decision factors used to screen the various CAAs, and develops and evaluates a set of selected CAAs that will meet the corrective action objectives.

All CAAs for CAU 574 are based on the presumption that all areas within the current NNSS boundary will be controlled in perpetuity and restricted from release to the public. As such, only industrial activities are permitted, and risks to receptors under residential scenarios will not be considered. Should the control of the NNSS change in the future to include public access or residential use, the selected CAAs may need to be reconsidered.

\section{B.1.1 CoRrective Action ObJectives}

On May 1, 1996, EPA issued an Advance Notice of Proposed Rulemaking (ANPR) for corrective action for releases from solid waste management units at hazardous waste management facilities (EPA, 1996). The EPA states that the ANPR should be considered the primary corrective action implementation guidance (Laws and Herman, 1997). The ANPR states that a basic operating principle for remedy selection is that corrective action decisions should be based on risk. It emphasizes that current and reasonably expected future land use should be considered when selecting corrective action remedies and encourages use of innovative site characterization techniques to expedite site investigations.

The ANPR provides the following EPA expectations for corrective action remedies (EPA, 1996):

- Treatment should be used to address principal threats wherever practicable and cost effective.

- Engineering controls, such as containment, should be used where wastes and contaminated media can be reliably contained, pose relatively low long-term threats, or for which treatment is impracticable.

- A combination of methods (e.g., treatment, engineering, and institutional controls) should be used, as appropriate, to protect human health and the environment.

- Institutional controls should be used primarily to supplement engineering controls as appropriate for short- or long-term management to prevent or limit exposure.

- Innovative technologies should be considered where such technologies offer potential for comparable or superior performance or implementability, less adverse impacts, or lower costs.

- Usable groundwater should be returned to maximum beneficial use wherever practicable.

- Contaminated soils should be remediated as necessary to prevent or limit direct exposure and to prevent the transfer of unacceptable concentrations of contaminants from soils to other media.

Implementation of the corrective action will ensure that contaminants remaining at each release site will not pose an unacceptable risk to human health and the environment, and that conditions at each site are in compliance with all applicable laws and regulations. 


\section{B.1.2 SCREENING CRITERIA}

The screening criteria used to evaluate and select the preferred CAA are identified in the Guidance on RCRA Corrective Action Decision Documents (EPA, 1991) and the Final RCRA Corrective Action Plan (EPA, 1994). CAAs are evaluated based on four general corrective action standards and five remedy selection decision factors. All CAAs must meet the four general standards to be selected for evaluation using the remedy selection decision factors.

The general corrective action standards are as follows:

- Protection of human health and the environment

- Compliance with media cleanup standards

- Control the source(s) of the release

- Comply with applicable federal, state, and local standards for waste management

The remedy selection decision factors are as follows:

- Short-term reliability and effectiveness

- Reduction of toxicity, mobility, and/or volume

- Long-term reliability and effectiveness

- Feasibility

- Cost

\section{B.1.3 CORRECTIVE ACTION STANDARDS}

The following subsections describe the corrective action standards used to evaluate the CAAs.

\section{Protection of Human Health and the Environment}

Protection of human health and the environment is a general mandate of the Resource Conservation and Recovery Act (RCRA) statute (EPA, 1994). This mandate requires that the corrective action include any necessary protective measures necessary to ensure the requirements are met. These measures may or may not be directly related to media cleanup, source control, or management of wastes.

\section{Compliance with Media Cleanup Standards}

The CAAs are evaluated for the ability to meet the proposed media cleanup standards. The media cleanup standards are the FALs.

\section{Control the Source(s) of the Release}

The CAAs are evaluated for the ability to stop further environmental degradation by controlling or eliminating additional releases that may pose a threat to human health and the environment. Unless source control measures are taken, efforts to clean up releases may be ineffective or, at best, will involve a perpetual cleanup. Therefore, each CAA must provide effective source control to ensure the long-term effectiveness and protectiveness of the corrective action.

\section{Comply with Applicable Federal, State, and Local Standards for Waste Management}

The CAAs are evaluated for the ability to be conducted in accordance with applicable federal and state regulations (e.g., 40 CFR 260 to 282, "Hazardous Waste Management” [CFR, 2010a]; 
40 CFR 761 “Polychlorinated Biphenyls,” [CFR, 2010b]; and Nevada Administrative Code (NAC) 444.842 to 980, "Facilities for Management of Hazardous Waste” [NAC, 2008]).

\section{B.1.4 REMEDY SELECTION DECISION FACTORS}

The following text describes the remedy selection decision factors used to evaluate the CAAs.

\section{Short-Term Reliability and Effectiveness}

Each CAA is evaluated with respect to its effects on human health and the environment during implementation of the selected corrective action. The following factors are addressed for each alternative:

- Protection of the community from potential risks associated with implementation (e.g., fugitive dusts, transportation of hazardous materials, and explosion)

- Protection of workers during implementation

- Adverse environmental impacts that may result from implementation

- The amount of time until the corrective action objectives are achieved

\section{Reduction of Toxicity, Mobility, and/or Volume}

Each CAA is evaluated for its ability to reduce toxicity, mobility, and/or volume of contaminated media, which refers to changes in one or more characteristics of the contaminated media by using corrective measures that decrease the inherent threats associated with that media.

\section{Long-Term Reliability and Effectiveness}

Each CAA is evaluated in terms of remaining risk after implementation of the CAA. The primary focus of this evaluation is on the extent and effectiveness of the control that may be required to manage the risk posed by treatment of residuals and/or untreated wastes.

\section{Feasibility}

Each CAA is evaluated for its technical and administrative feasibility and the availability of services and materials needed during implementation. The following criteria are evaluated:

- Construction and Operation - The feasibility of implementing a CAA given existing waste and site-specific conditions

- Administrative Feasibility - The administrative activities needed to implement the CAA (e.g., permits, URs, public acceptance, rights of way, and offsite approval)

- Availability of Services and Materials - The availability of adequate treatment, storage capacity, disposal services, necessary technical services and materials, and prospective technologies for each CAA

\section{Cost}

Costs for each alternative are estimated for comparison purposes only. The cost estimate for each CAA includes both capital, and operation and maintenance costs, as applicable. 


\section{B.1.5 DeVelopment of CoRrective ACtion Alternatives}

Date: August 2011

This section identifies and briefly describes the viable corrective action technologies and the CAAs considered for NEPTUNE and BLANCA. Contamination providing a dose exceeding the FAL was assumed to be present in subsurface soils at NEPTUNE and BLANCA.

Based on a review of existing data, future use, and current operations at the NNSS, the following alternatives have been developed for consideration at CAU 574:

- Alternative 1 - No Further Action

- Alternative 2 - Clean Closure

- Alternative 3 - Closure in Place

\section{B.1.5.1 Alternative 1 - No Further Action}

Under the no further action alternative, no corrective action activities will be implemented. This alternative is a baseline case with which to compare and assess the other CAAs and their ability to meet the corrective action standards.

\section{B.1.5.2 Alternative 2 - Clean Closure}

Alternative 2 includes excavating and disposing of impacted soil and debris presenting a dose exceeding the FAL to a depth of $25 \mathrm{ft}$ bgs (the maximum depth to which a construction activity might excavate for a building foundation or basement). Verification soil samples will be collected and analyzed for the presence of a dose exceeding the FAL following removal of contaminated soil. Contaminated materials removed will be disposed at an appropriate disposal facility. Excavated areas will be returned to surface conditions compatible with the intended future use of the site.

\section{B.1.5.3 Alternative 3 - Closure in Place}

Alternative 3 includes the implementation of a UR where a radiological dose is present at levels that exceed the FAL. This UR will restrict inadvertent contact with contaminated media by prohibiting any activity that would cause a site worker to be exposed to a dose exceeding $25 \mathrm{mrem} / \mathrm{yr}$. Under this alternative, debris within the FAL area will not be removed.

\section{B.1.6 EVALUATION AND COMPARISON OF Alternatives}

Each CAA presented in Section B.1.5 will be evaluated based on the general corrective action standards listed in Section B.1.2. This evaluation is presented in Table B.1. Any CAA that does not meet the general corrective action standards will be removed from consideration.

Only CAAs 2 and 3 meet all corrective action standards and will be further evaluated based on the remedy selection decision factors described in Section B.1.4. This evaluation is presented in Table B.2. For each remedy selection decision factor, the CAAs are ranked relative to one another. The CAA with the least desirable impact on the remedy selection decision factor will be given a ranking of 1 . The CAAs with increasingly desirable impacts on the remedy selection decision factor will receive increasing rank numbers. The CAAs that will have an equal impact on the remedy selection decision factor will receive an equal ranking number. The scoring listed in Table B.2 represents the sum of the remedy selection decision factor rankings for each CAA. 
TABle B.1. Evaluation of General Corrective ACtion Standards

\begin{tabular}{|c|c|c|}
\hline \multicolumn{3}{|c|}{$\begin{array}{l}\text { CAS 12-23-10, U12c.03 Crater (Neptune) } \\
\text { and CAS 12-45-01, U12e.05 Crater (Blanca) }\end{array}$} \\
\hline \multicolumn{3}{|c|}{ CAA 1, No Further Action } \\
\hline Standard & Comply? & Explanation \\
\hline Protection of Human Health and the Environment & No & $\begin{array}{l}\text { Contamination is present that could provide a worker } \\
\text { dose exceeding the FAL. }\end{array}$ \\
\hline Compliance with Media Cleanup Standards & No & $\begin{array}{l}\text { Contamination is present that could provide a worker } \\
\text { dose exceeding the FAL. }\end{array}$ \\
\hline Control the Source(s) of the Release & Yes & $\begin{array}{l}\text { The sources of the releases were singular events and do } \\
\text { not continue to release additional contamination. }\end{array}$ \\
\hline $\begin{array}{l}\text { Comply with Applicable Federal, State, and Local } \\
\text { Standards for Waste Management }\end{array}$ & Yes & This alternative will not generate waste. \\
\hline \multicolumn{3}{|c|}{ CAA 2, Clean Closure } \\
\hline Standard & Comply? & Explanation \\
\hline Protection of Human Health and the Environment & Yes & Contamination exceeding the FAL will be removed. \\
\hline Compliance with Media Cleanup Standards & Yes & Contamination exceeding the FAL will be removed. \\
\hline Control the Source(s) of the Release & Yes & Contamination exceeding the FAL will be removed. \\
\hline $\begin{array}{l}\text { Comply with Applicable Federal, State, and Local } \\
\text { Standards for Waste Management }\end{array}$ & Yes & $\begin{array}{l}\text { Excavated waste can be managed in compliance with all } \\
\text { standards. }\end{array}$ \\
\hline \multicolumn{3}{|c|}{ CAA 3, Closure in Place with Administrative Controls } \\
\hline Standard & Comply? & Explanation \\
\hline Protection of Human Health and the Environment & Yes & $\begin{array}{l}\text { A UR will be implemented to protect workers from } \\
\text { inadvertent exposure. }\end{array}$ \\
\hline Compliance with Media Cleanup Standards & Yes & $\begin{array}{l}\text { Although contamination will not be removed, the site } \\
\text { will be controlled to prevent workers from receiving a } \\
\text { dose exceeding } 25 \mathrm{mrem} / \mathrm{yr} \text {. }\end{array}$ \\
\hline Control the Source(s) of the Release & Yes & $\begin{array}{l}\text { The sources of the releases were singular events and do } \\
\text { not continue to release additional contamination. }\end{array}$ \\
\hline $\begin{array}{l}\text { Comply with Applicable Federal, State, and Local } \\
\text { Standards for Waste Management }\end{array}$ & Yes & This alternative will not generate waste. \\
\hline
\end{tabular}


TABLE B.2. EVALUATION OF REMEDY SELECTION DECISION FACTORS

\begin{tabular}{|c|c|c|}
\hline \multicolumn{3}{|r|}{$\begin{array}{l}\text { CAS 12-23-10, U12c.03 Crater (Neptune) } \\
\text { and CAS 12-45-01, U12e.05 Crater (Blanca) }\end{array}$} \\
\hline \multicolumn{3}{|r|}{ CAA 1, No Further Action } \\
\hline \multicolumn{3}{|c|}{ Not evaluated, as this CAA did not meet the General Corrective Action Standards } \\
\hline \multicolumn{3}{|r|}{ CAA 2, Clean Closure } \\
\hline Standard & Rank & Explanation \\
\hline $\begin{array}{l}\text { Short-Term Reliability } \\
\text { and Effectiveness }\end{array}$ & 1 & $\begin{array}{l}\text { This alternative is reliable and effective, but involves high short-term risk to personnel } \\
\text { working on steep slopes during soil removal operations. }\end{array}$ \\
\hline $\begin{array}{l}\text { Reduction of Toxicity, } \\
\text { Mobility, and/or Volume }\end{array}$ & 2 & $\begin{array}{l}\text { This alternative will result in a decrease of toxicity and mobility, but will generate significant } \\
\text { waste volumes. }\end{array}$ \\
\hline $\begin{array}{l}\text { Long-Term Reliability } \\
\text { and Effectiveness }\end{array}$ & 2 & $\begin{array}{l}\text { This alternative is reliable and effective at protecting human health and the environment } \\
\text { because removal of the contaminated media will eliminate future exposure of site workers to } \\
\text { contamination. }\end{array}$ \\
\hline Feasibility & 1 & $\begin{array}{l}\text { This option would involve the excavation and disposal of more than } 310,000 \text { cubic meters of } \\
\text { soil on steep slopes in a remote location. }\end{array}$ \\
\hline Cost & 1 & Cost is estimated to be in excess of $\$ 100$ million. \\
\hline Score & 7 & \\
\hline \multicolumn{3}{|r|}{ CAA 3, Closure in Place with Administrative Controls } \\
\hline Standard & Rank & Explanation \\
\hline $\begin{array}{l}\text { Short-Term Reliability } \\
\text { and Effectiveness }\end{array}$ & 2 & $\begin{array}{l}\text { This alternative is reliable and effective in providing increased protection of human health by } \\
\text { minimizing work on steep slopes and avoiding contact with contamination. }\end{array}$ \\
\hline $\begin{array}{l}\text { Reduction of Toxicity, } \\
\text { Mobility, and/or Volume }\end{array}$ & 1 & $\begin{array}{l}\text { This alternative will not reduce toxicity or mobility of the contaminants that are present, but } \\
\text { will not generate excavation waste volumes. }\end{array}$ \\
\hline $\begin{array}{l}\text { Long-Term Reliability } \\
\text { and Effectiveness }\end{array}$ & 1 & $\begin{array}{l}\text { This alternative is reliable in the long term with ongoing maintenance. It is effective in } \\
\text { providing protection of human health by preventing inadvertent contact with contamination. }\end{array}$ \\
\hline Feasibility & 2 & $\begin{array}{l}\text { This alternative is easily implemented and would not require significant additional } \\
\text { maintenance and long-term monitoring. }\end{array}$ \\
\hline Cost & 2 & $\begin{array}{l}\text { The installation costs are estimated at } \$ 25,000 \text {. Ongoing maintenance costs for this } \\
\text { alternative are estimated at } \$ 1,000 \text { annually. }\end{array}$ \\
\hline Score & 8 & \\
\hline
\end{tabular}




\section{B.2.0 RECOMMENDED ALTERNATIVE}

Three CAAs were evaluated for NEPTUNE and BLANCA. These include no further action (CAA 1), clean closure (CAA 2), and closure in place (CAA 3). Only CAA 2 and CAA 3 met all requirements for general corrective action standards (Section B.1.3). In general, for the clean closure alternative, near-surface soils would be removed from the sites to a depth of $25 \mathrm{ft}$ bgs. For the closure in place alternative, potential worker exposure to radiological contamination would be controlled through the implementation of URs. Both CAAs would, therefore, be protective of human health and the environment, comply with media cleanup standards, and control the source of release. As supported by the following discussion, further examination of the two CAAs by the five EPA remedy selection decision factors resulted in the selection of closure in place as the preferred CAA for both NEPTUNE and BLANCA.

While clean closure is both reliable and effective in the long term, this alternative is not feasible at these sites due to the steep slopes and remoteness of the sites. In contrast, closure in place provides protection of inadvertent worker exposures by restricting the use of the sites. The usefulness of these sites for any construction or work activities is also very limited as the steep hillsides are not amenable to these activities.

Based upon the five remedy selection decision factors, clean closure received an overall score of 7 (less desirable), whereas closure in place received an overall score of 8 (more desirable). This result was not only the product of an examination of the two CAAs by the five remedy selection decision factors, but also in consideration of the current NNSS administrative controls (e.g., NNSS access restrictions and control of site activities), the remoteness of the sites, no nearby structures or activities, no current or planned use of the sites, and the steep hillside location. Therefore, the significant safety risks and expense required to clean close these sites would not result in significant additional protection to potential future receptors.

Therefore, selection of the CAA of closure in place for both NEPTUNE and BLANCA is consistent with past practices for CASs that contain contamination above the FAL and where there would be significant costs and significant short-term risks to workers involved in cleanup activities. However, if the land use at the NNSS should change in the future to include public access or residential use, the selected CAA may need to be reconsidered. 


\section{B.3.0 REFERENCES}

CFR, see Code of Federal Regulations.

Code of Federal Regulations, 2010a. Title 40 CFR Parts 260 to 282, "Hazardous Waste Management.” Washington, D.C.

Code of Federal Regulations, 2010b. Title 40 Part CFR 761, "Polychlorinated Biphenyls (PCBs) Manufacturing, Processing, Distribution in Commerce, and Use Prohibitions.” Washington, D.C.

EPA, see U.S. Environmental Protection Agency.

Laws, E. P., and S. A. Herman, U.S. Environmental Protection Agency, 1997. Memorandum to RCRA/CERCLA Senior Policy Managers Region I-X titled "Use of the Corrective Action Advance Notice of Proposed Rulemaking as Guidance,” 17 January. Washington, D.C.

NAC, see Nevada Administrative Code.

NSTec, see National Security Technologies, LLC.

Nevada Administrative Code, 2008. NAC 444.842 to 444.980, “Facilities for Management of Hazardous Waste.” Carson City, NV. As accessed at http://www.leg.state.nv.us/nac on July 1, 2011.

U.S. Environmental Protection Agency, 1991. Guidance on RCRA Corrective Action Decision Documents: The Statement of Bases, Final Decision and Response to Comments. EPA/540/G-91/011. Washington, D.C.

U.S. Environmental Protection Agency, 1994. Final RCRA Corrective Action Plan. EPA/520-R-94-004. Washington, D.C.

U.S. Environmental Protection Agency, 1996. “Corrective Action for Releases from Solid Waste Management Units at Hazardous Waste Management Facilities,” 1 May. In Federal Register, Vol. 61, No. 85. Washington, D.C. 
Revision: 0

Date: August 2011

\section{LIBRARY DISTRIBUTION LIST}


Revision: 0

Date: August 2011

\section{THIS PAGE INTENTIONALLY LEFT BLANK}




\section{LIBRARY DISTRIBUTION LIST}

U.S. Department of Energy

National Nuclear Security Administration

Nevada Site Office

Technical Library

P.O. Box 98518, M/S 505

Las Vegas, NV 89193-8518

U.S. Department of Energy

Office of Scientific and Technical Information

P.O. Box 62

Oak Ridge, TN 37831-0062

Southern Nevada Public Reading Facility

c/o Nuclear Testing Archive

P.O. Box 98521, M/S 400

Las Vegas, NV 89193-8521

Manager, Northern Nevada FFACO

Public Reading Facility

c/o Nevada State Library \& Archives

Carson City, NV 89701-4285
1 (Uncontrolled, electronic copy)

1 (Uncontrolled, electronic copy)

2 (Uncontrolled, electronic copies)

1 (Uncontrolled, electronic copy) 
Revision: 0

Date: August 2011

\section{THIS PAGE INTENTIONALLY LEFT BLANK}

